\title{
MULTIVARIABLE LUBIN-TATE $(\varphi, \Gamma)$-MODULES AND FILTERED $\varphi$-MODULES
}

\section{LAURENT BERGER}

\begin{abstract}
We define some rings of power series in several variables, that are attached to a Lubin-Tate formal module. We then give some examples of $(\varphi, \Gamma)$-modules over those rings. They are the global sections of some reflexive sheaves on the $p$-adic open unit polydisk, that are constructed from a filtered $\varphi$-module using a modification process. We prove that we obtain every crystalline $(\varphi, \Gamma)$-module over those rings in this way.
\end{abstract}

\section{Contents}

Introduction

1. Periods of Lubin-Tate formal groups

2. Rings of multivariable power series

3. Embeddings in $\mathbf{B}_{\mathrm{dR}}$

4. $\left(\varphi_{q}, \Gamma_{F}\right)$-modules in one variable 416

5. Construction of $\mathcal{R}^{+}(Y)$-modules $\quad 419$

6. Properties of $\mathrm{M}^{+}(D) \quad 421$

7. Crystalline $\left(\varphi_{q}, \Gamma_{F}\right)$-modules 424

$\begin{array}{ll}\text { Acknowledgments } & 427\end{array}$

$\begin{array}{ll}\text { References } & 427\end{array}$

\section{Introduction}

Let $F$ be the unramified extension of $\mathbf{Q}_{p}$ of degree $h$ and let $q=p^{h}$ so that the residue field of $\mathcal{O}_{F}$ is $\mathbf{F}_{q}$. We fix an embedding $F \subset \overline{\mathbf{Q}}_{p}$ so that if $\sigma: F \rightarrow F$ denotes the absolute Frobenius map, which lifts $x \mapsto x^{p}$ on $\mathbf{F}_{q}$, then the $h$ embeddings of $F$ into $\overline{\mathbf{Q}}_{p}$ are given by Id, $\sigma, \ldots, \sigma^{h-1}$. The symbol $\varphi_{q}$ denotes a $\sigma^{h}$-semilinear Frobenius map. If $K$ is a subfield of $\overline{\mathbf{Q}}_{p}$, then let $G_{K}=\operatorname{Gal}\left(\overline{\mathbf{Q}}_{p} / K\right)$.

The goal of this article is to present a first attempt at constructing some "multivariable Lubin-Tate $(\varphi, \Gamma)$-modules," that is some $\left(\varphi_{q}, \Gamma_{F}\right)$-modules over rings of power series in $h$ variables, on which $\Gamma_{F}=\mathcal{O}_{F}^{\times}$acts by a formula arising from a Lubin-Tate formal $\mathcal{O}_{F}$-module. A construction of such $\left(\varphi_{q}, \Gamma_{F}\right)$-modules, but "in one variable," was carried out by Kisin and Ren in [18]: they prove that in certain cases, the $\left(\varphi_{q}, \Gamma_{F}\right)$ modules arising from Fontaine's standard construction of [12] are overconvergent.

Received by the editors April 29, 2013.

1991 Mathematics Subject Classification. 11F; 11S; 14G.

Key words and phrases. $(\varphi, \Gamma)$-module; Lubin-Tate group; filtered $\varphi$-module; crystalline representation; $p$-adic period; Fontaine theory; reflexive sheaf. 
In order to do so, Kisin and Ren adapt the construction of $(\varphi, \Gamma)$-modules attached to filtered $(\varphi, N)$-modules given in [4] to their setting, which allows them to attach a $\left(\varphi_{q}, \Gamma_{F}\right)$-module in one variable to a filtered $\varphi_{q}$-module. They then point out in the introduction of [18] that "it seems likely that in order to obtain a classification valid for any crystalline $G_{K}$-representation one needs to consider higher dimensional subrings of $W(\operatorname{Fr} R)$, constructed using the periods of all the conjugates of [the Lubin-Tate group]."

The motivation for these computations is the hope that we can construct some representations of the Borel subgroup of $\mathrm{GL}_{2}(F)$, for example using the recipe given by Colmez in [11], that would shed some light on the $p$-adic local Langlands correspondence for $\mathrm{GL}_{2}(F)$ (see [9]). Theorems A, B and C below are a very first step in this direction, but remain insufficient. In particular, the " $p$-adic Fourier theory" of Schneider and Teitelbaum (see [20]) will very likely play an important role in the sequel.

We now describe our results in more detail. Let $\mathrm{LT}_{h}$ be the Lubin-Tate formal $\mathcal{O}_{F}$-module for which multiplication by $p$ is given by $[p](T)=p T+T^{q}$. We denote by $[a](T)$ the element of $\mathcal{O}_{F} \llbracket T \rrbracket$ that gives the action of $a \in \mathcal{O}_{F}$ on $\mathrm{LT}_{h}$. We consider two rings $\mathcal{R}^{+}(Y)$ and $\mathcal{R}(Y)$ of power series in the $h$ variables $Y_{0}, \ldots, Y_{h-1}$, with coefficients in $F$. The ring $\mathcal{R}^{+}(Y)$ is the ring of power series that converge on the open unit polydisk, and $\mathcal{R}(Y)$ is the Robba ring that corresponds to it, by adapting Schneider's construction given in the appendix of [23]. The action of the group $\mathcal{O}_{F}^{\times}$ on those rings is given by the formula $a\left(Y_{j}\right)=\left[\sigma^{j}(a)\right]\left(Y_{j}\right)$, and the Frobenius map $\varphi_{q}$ is given by $\varphi_{q}\left(Y_{j}\right)=[p]\left(Y_{j}\right)$.

The construction of $p$-adic periods for Lubin-Tate groups gives rise to a map $\mathcal{R}^{+}(Y) \rightarrow \widetilde{\mathbf{B}}_{\text {rig }}^{+}$, where $\widetilde{\mathbf{B}}_{\text {rig }}^{+}$is the Fréchet completion of $\widetilde{\mathbf{B}}^{+}=W\left(\widetilde{\mathbf{E}}^{+}\right)[1 / p]$, and we prove (Corollary 3.7) that this map is in fact injective (remark: if $\widetilde{\mathcal{R}}^{+}(Y)$ denotes the completion of the perfection of $\mathcal{R}^{+}(Y)$, then the map above extends to a map $\widetilde{\mathcal{R}}^{+}(Y) \rightarrow \widetilde{\mathbf{B}}_{\text {rig }}^{+}$but note that, by the theory of the field of norms of $[14,22]$, this latter map is not injective anymore if $h \geqslant 2$. This has prevented us from studying étale $\varphi_{q}$-modules using Kedlaya's methods, so such considerations are absent from this paper).

Let $D$ be a finite dimensional $F$-vector space, endowed with an $F$-linear Frobenius map $\varphi_{q}: D \rightarrow D$, and an action of $G_{F}$ on $D$ that factors through $\Gamma_{F}$ and commutes with $\varphi_{q}$. For each $0 \leqslant j \leqslant h-1$, let Fil $_{j}^{\bullet}$ be a filtration on $F \otimes_{F}^{\sigma^{j}} D \simeq D$ that is stable under $\Gamma_{F}$.

For example, if $V$ is an $F$-linear crystalline representation of $G_{F}$ of dimension $d$, then $\mathrm{D}_{\text {cris }}(V)$ is a free $F \otimes \mathbf{Q}_{p} F$-module of rank $d$, and we have

$$
\mathrm{D}_{\text {cris }}(V)=D \oplus \varphi(D) \oplus \cdots \oplus \varphi^{h-1}(D),
$$

according to the decomposition of $F \otimes_{\mathbf{Q}_{p}} F$ as $\prod_{\sigma^{i}: F \rightarrow F} F$. Each $\varphi^{j}(D)$ has the filtration induced from $\mathrm{D}_{\text {cris }}(V)$, and we set $\operatorname{Fil}_{j}^{k} D=\varphi^{-j}\left(\operatorname{Fil}^{k} \mathrm{D}_{\text {cris }}(V) \cap \varphi^{j}(D)\right)$.

The composite of the map $\mathcal{R}^{+}(Y) \rightarrow \widetilde{\mathbf{B}}_{\text {rig }}^{+}$with the map $\varphi^{-k}: \widetilde{\mathbf{B}}_{\text {rig }}^{+} \rightarrow \widetilde{\mathbf{B}}_{\text {rig }}^{+}$gives rise to a map $\iota_{k}: \mathcal{R}^{+}(Y) \rightarrow \widetilde{\mathbf{B}}_{\text {rig }}^{+}$. Let $\log _{\mathrm{LT}}(T)$ be the logarithm of $\mathrm{LT}_{h}$, and let $\lambda_{j}=\log _{\mathrm{LT}}\left(Y_{j}\right) / Y_{j}$ and $\lambda=\lambda_{0} \times \cdots \times \lambda_{h-1}$ (note that the image of $\prod_{j=0}^{h-1} \log _{\mathrm{LT}}\left(Y_{j}\right)$ in 
$\widetilde{\mathbf{B}}_{\text {rig }}^{+}$is some $\mathbf{Q}_{p}$-multiple of $t=\log (1+X)$, so that $\lambda$ is an analogue of $\left.t / X\right)$. Define

$$
\mathrm{M}^{+}(D)=\left\{y \in \mathcal{R}^{+}(Y)[1 / \lambda] \otimes_{F} D, \iota_{k}(y) \in \mathrm{Fil}_{-k}^{0}\left(\mathbf{B}_{\mathrm{dR}} \otimes_{F}^{\sigma^{-k}} D\right) \text { for all } k \geqslant h\right\} .
$$

The ring $\mathcal{R}^{+}(Y)$ is a Fréchet-Stein algebra in the sense of [21], and we therefore have the notion of coadmissible $\mathcal{R}^{+}(Y)$-modules, which are the global sections of coherent sheaves on the open unit polydisk.

Theorem A. The module $\mathrm{M}^{+}(D)$ is a reflexive coadmissible $\mathcal{R}^{+}(Y)$-module, for all $0 \leqslant j \leqslant h-1, \mathrm{M}^{+}(D)\left[\lambda_{j} / \lambda\right]$ is a free $\mathcal{R}^{+}(Y)\left[\lambda_{j} / \lambda\right]$-module of rank $d$, and we have $\mathrm{M}^{+}(D)=\cap_{j=0}^{h-1} \mathrm{M}^{+}(D)\left[\lambda_{j} / \lambda\right]$.

The definition of $\mathrm{M}^{+}(D)$ is analogous to the one given in $[4,18]$ and similar articles. When $h=1$, the proof of theorem A relies on the fact that $\mathrm{M}^{+}(D)$ can be seen as a vector bundle on the open unit disk. Our proof of theorem A relies on the one dimensional case, and on the interpretation of $\mathrm{M}^{+}(D)$ as the global sections of a coherent sheaf on the open unit polydisk.

Remark. If $h \leqslant 2$, then $\mathcal{R}^{+}(Y)$ is of dimension $\leqslant 2$ and one can then prove that $\mathrm{M}^{+}(D)$, being reflexive, is actually free of rank $d$ (see Remark 5.7). If $h \geqslant 3$, I do not know whether $\mathrm{M}^{+}(D)$ is free of rank $d$ in general.

Let $\mathrm{M}(D)=\mathcal{R}(Y) \otimes_{\mathcal{R}^{+}(Y)} \mathrm{M}^{+}(D)$, so that $\mathrm{M}(D)$ is a $\left(\varphi_{q}, \Gamma_{F}\right)$-module over the multivariable Robba ring $\mathcal{R}(Y)$ (see Definition 6.4).

Theorem B. If $V$ is an F-linear crystalline representation of $G_{F}$, and if $D$ arises from $\mathrm{D}_{\text {cris }}(V)$ as above, then there is a natural map $\widetilde{\mathbf{B}}_{\text {rig }}^{\dagger} \otimes_{\mathcal{R}(Y)} \mathrm{M}(D) \rightarrow \widetilde{\mathbf{B}}_{\text {rig }}^{\dagger} \otimes_{F} V$, and this map is an isomorphism.

If $\mathrm{M}$ is a $\left(\varphi_{q}, \Gamma_{F}\right)$-module over $\mathcal{R}(Y)$, then we set $\mathrm{D}_{\text {cris }}(\mathrm{M})=\left(\mathcal{R}(Y)[1 / t] \otimes_{\mathcal{R}(Y)}\right.$ $\mathrm{M})^{\Gamma_{F}}$, and we say that $\mathrm{M}$ is crystalline if (1) $\mathrm{M}\left[\lambda_{j} / \lambda\right]$ is a free $\mathcal{R}(Y)\left[\lambda_{j} / \lambda\right]$-module of some rank $d$ for all $j,(2) \mathrm{M}=\cap_{j=0}^{h-1} \mathrm{M}\left[\lambda_{j} / \lambda\right]$, and (3) $\operatorname{dim} \mathrm{D}_{\text {cris }}(\mathrm{M})=d$. For example, if $D$ is a filtered $\varphi_{q}$-module with $h$ filtrations Fil $_{j}^{\bullet}$ as above, on which the action of $\Gamma_{F}$ is trivial, then $\mathrm{M}(D)$ is a crystalline $\left(\varphi_{q}, \Gamma_{F}\right)$-module.

Theorem C. The functors $\mathrm{M} \mapsto \mathrm{D}_{\text {cris }}(\mathrm{M})$ and $D \mapsto \mathrm{M}(D)$, between the category of crystalline $\left(\varphi_{q}, \Gamma_{F}\right)$-modules over $\mathcal{R}(Y)$ and the category of $\varphi_{q}$-modules with $h$ filtrations, are mutually inverse.

Note that if $h=1$, then the $(\varphi, \Gamma)$-modules that we construct are the classical cyclotomic ones, and theorems A, B and $\mathrm{C}$ are well-known.

We now give a short description of the contents of this paper: in Section 1, we give some reminders about the $p$-adic periods of Lubin-Tate formal $\mathcal{O}_{F}$-modules. In Section 2, we define the various rings of power series that we use, and establish some of their properties. In Section 3, we embed those rings in the usual rings of $p$-adic periods. In Section 4 , we briefly survey Kisin and Ren's construction and explain why $\left(\varphi_{q}, \Gamma_{F}\right)$ modules over rings of power series in several variables are needed. In Section 5, we attach such objects to filtered $\varphi_{q}$-modules and prove Theorem A. In Section 6, we define $\left(\varphi_{q}, \Gamma_{F}\right)$-modules and prove Theorem B. In Section 7 , we study crystalline $\left(\varphi_{q}, \Gamma_{F}\right)$-modules and prove Theorem $\mathrm{C}$. 


\section{Periods of Lubin-Tate formal groups}

Let $\mathrm{LT}_{h}$ be the Lubin-Tate formal $\mathcal{O}_{F}$-module for which multiplication by $p$ is given by $[p](T)=p T+T^{q}$. We denote by $[a](T)$ the element of $\mathcal{O}_{F} \llbracket T \rrbracket$ that gives the action of $a \in \mathcal{O}_{F}$ on $\mathrm{LT}_{h}$ and by $S(T, U)=T \oplus U$ the element of $\mathcal{O}_{F} \llbracket T, U \rrbracket$ that gives addition.

Let $\pi_{0}=0$ and for each $n \geqslant 1$, let $\pi_{n} \in \overline{\mathbf{Q}}_{p}$ be such that $[p]\left(\pi_{n}\right)=\pi_{n-1}$, with $\pi_{1} \neq 0$. We have $\operatorname{val}_{p}\left(\pi_{n}\right)=1 / q^{n-1}(q-1)$ if $n \geqslant 1$. Let $F_{n}=F\left(\pi_{n}\right)$ and let $F_{\infty}=\cup_{n \geqslant 1} F_{n}$. Recall that $\operatorname{Gal}\left(F_{\infty} / F\right) \simeq \mathcal{O}_{F}^{\times}$and that the maximal abelian extension of $F$ is $F_{\infty} \cdot F^{\text {unr }}$. Denote by $H_{F}$ the group $\operatorname{Gal}\left(\overline{\mathbf{Q}}_{p} / F_{\infty}\right)$, by $\Gamma_{F}$ the $\operatorname{group} \operatorname{Gal}\left(F_{\infty} / F\right)$ and by $\chi_{\mathrm{LT}}$ the isomorphism $\chi_{\mathrm{LT}}: \Gamma_{F} \rightarrow \mathcal{O}_{F}^{\times}$. In the sequel, we sometimes directly identify $\Gamma_{F}$ with $\mathcal{O}_{F}^{\times}$, that is we drop " $\chi_{\mathrm{LT}}$ " from the notation to make the formulas less cumbersome.

Let $\widetilde{\mathbf{E}}^{+}=\varliminf_{x \mapsto x^{q}} \mathcal{O}_{\mathbf{C}_{p}} / p$ and $\widetilde{\mathbf{A}}^{+}=W\left(\widetilde{\mathbf{E}}^{+}\right)$denote Fontaine's rings of periods (see [13]). Note that we take the limit with respect to the maps $x \mapsto x^{q}$, which does not change the rings. Let $\varphi_{q}: \widetilde{\mathbf{A}}^{+} \rightarrow \widetilde{\mathbf{A}}^{+}$be given by $\varphi_{q}=\varphi^{h}$. Recall that in Section 9.2 of [10], Colmez has constructed a map $\{\cdot\}: \widetilde{\mathbf{E}}^{+} \rightarrow \widetilde{\mathbf{A}}^{+}$having the following property: if $x \in \widetilde{\mathbf{E}}^{+}$, then $\{x\}$ is the unique element of $\widetilde{\mathbf{A}}^{+}$that lifts $x$ and satisfies $\varphi_{q}(\{x\})=[p](\{x\})$. Let $\theta: \widetilde{\mathbf{A}}^{+} \rightarrow \mathcal{O}_{\mathbf{C}_{p}}$ denote Fontaine's map (see [13]). If $x=\left(x_{0}, x_{1}, \ldots\right)$, then $\theta(\{x\})=\lim _{n \rightarrow \infty}\left[p^{n}\right]\left(\widehat{x}_{n}\right)$, where $\widehat{x}_{n} \in \mathcal{O}_{\mathbf{C}_{p}}$ is any lift of $x_{n}$.

Let $u=\left\{\left(\bar{\pi}_{0}, \bar{\pi}_{1}, \ldots\right)\right\} \in \widetilde{\mathbf{A}}^{+}$, so that $g(u)=\left[\chi_{\mathrm{LT}}(g)\right](u)$ if $g \in G_{F}$.

Let $\log _{\mathrm{LT}}(T) \in F \llbracket T \rrbracket$ denote the Lubin-Tate logarithm map, which converges on the open unit disk and satisfies $\log _{\mathrm{LT}}([a](T))=a \cdot \log _{\mathrm{LT}}(T)$ if $a \in \mathcal{O}_{F}$. Recall (see Section 9.3 of [10]) that $\log _{\mathrm{LT}}(u)$ converges in $\widetilde{\mathbf{B}}_{\text {rig }}^{+}$to an element $t_{F}$ which satisfies $g\left(t_{F}\right)=\chi_{\mathrm{LT}}(g) \cdot t_{F}$.

Let $Q_{k}(T)$ be the minimal polynomial of $\pi_{k}$ over $F$. We have $Q_{0}(T)=T, Q_{1}(T)=$ $p+T^{q-1}$ and $Q_{k+1}(T)=Q_{k}([p](T))$ if $k \geqslant 1$. Note that $\log _{\mathrm{LT}}(T)=T \cdot \prod_{k \geqslant 1} Q_{k}(T) / p$. Indeed, $\log _{\mathrm{LT}}(T)=\lim _{k \rightarrow \infty} p^{-k} \cdot\left[p^{k}\right](T)$ (Section 9.3 of $[10]$ ) and $\left[p^{k}\right](T)=Q_{0}(T) \cdots$ $Q_{k}(T)$. Let $\exp _{\mathrm{LT}}(T)$ denote the inverse of $\log _{\mathrm{LT}}(T)$. We have $\exp _{\mathrm{LT}}(T)=\sum_{k=1}^{\infty} e_{k} T^{k}$ with $v_{p}\left(e_{k}\right) \geqslant-k /(q-1)$. For example, $\log _{\mathbf{G}_{\mathrm{m}}}(T)=\log (1+T)$ and $\exp _{\mathbf{G}_{\mathrm{m}}}(T)=$ $\exp (T)-1$.

Remark 1.1. Our special choice of $[p](T)=p T+T^{q}$ is the simplest. Since $[p](T)$ belongs to $\mathbf{Z}_{p}[T]$, the series $Q_{k}(T), \log _{\mathrm{LT}}(T)$ and $\exp _{\mathrm{LT}}(T)$ all have coefficients in $\mathbf{Q}_{p}$. It also implies that $[\sigma(a)](T)=\sigma([a](T))$, since $[a](T)=\exp _{\mathrm{LT}}\left(a \cdot \log _{\mathrm{LT}}(T)\right)$.

Lemma 1.2. If $z \in \mathfrak{m}_{\mathbf{C}_{p}}$, then

$$
\frac{[1+a](z)-z}{a}=\log _{\mathrm{LT}}(z) \cdot \frac{d S}{d U}(z, 0)+\mathrm{O}(a)
$$

as $a \rightarrow 0$ in $\mathcal{O}_{F}$.

Proof. We are looking at the limit of $(S(z,[a](z))-z) / a$ as $a \rightarrow 0$. If $a$ is small enough, then $[a](z)=\exp _{\mathrm{LT}}\left(a \cdot \log _{\mathrm{LT}}(z)\right)=a \cdot \log _{\mathrm{LT}}(z)+\mathrm{O}\left(a^{2}\right)$, which implies the lemma.

\section{Rings of multivariable power series}

We consider power series in the $h$ variables $Y_{0}, \ldots, Y_{h-1}$. If $Y^{m}=Y_{0}^{m_{0}} \ldots Y_{h-1}^{m_{h-1}}$ is a monomial, then its weight is $w(m)=m_{0}+p m_{1}+\cdots+p^{h-1} m_{h-1}$. If $I$ is a subinterval of 
$[0 ;+\infty]$ and if $J=\left\{j_{1}, \ldots, j_{k}\right\}$ is a subset of $\{0, \ldots, h-1\}$, then (adapting Appendix A of [23] to our situation) we define $\mathcal{R}^{I}\left(\left\{Y_{j}\right\}_{j \in J}\right)$ to be the ring of power series

$$
f\left(Y_{j_{1}}, \ldots, Y_{j_{k}}\right)=\sum_{m_{1}, \ldots, m_{k} \in \mathbf{Z}} a_{m_{1} \ldots m_{k}} Y_{j_{1}}^{m_{1}} \ldots Y_{j_{k}}^{m_{k}},
$$

such that $\operatorname{val}_{p}\left(a_{m}\right)+w(m) / r \rightarrow+\infty$ for all $r \in I$. In other words, $f(Y)$ is required to converge on the polyannulus $\left\{\left(Y_{0}, \ldots, Y_{h-1}\right)\right.$ such that $\left|Y_{0}\right|=p^{-1 / r}, \ldots,\left|Y_{h-1}\right|=$ $\left.p^{-p^{h-1} / r}\right\}$ for all $r \in I$. We then define $W(f(Y), r)=\inf _{m \in \mathbf{Z}}\left(\operatorname{val}_{p}\left(a_{m}\right)+w(m) / r\right)$ and, if $I$ is closed, $W(f(Y), I)=\inf _{r \in I} W(f(Y), r)$.

We let $\mathcal{R}^{+}\left(\left\{Y_{j}\right\}_{j \in J}\right)=\mathcal{R}^{[0 ;+\infty}\left[\left(\left\{Y_{j}\right\}_{j \in J}\right)\right.$ be the ring of holomorphic functions on the open unit polydisk corresponding to $J$. The Robba $\operatorname{ring} \mathcal{R}\left(\left\{Y_{j}\right\}_{j \in J}\right)$ is defined as $\mathcal{R}\left(\left\{Y_{j}\right\}_{j \in J}\right)=\cup_{r \geqslant 0} \mathcal{R}^{[r ;+\infty}\left[\left(\left\{Y_{j}\right\}_{j \in J}\right)\right.$. In order to lighten the notation, we write $\mathcal{R}^{I}(Y), \mathcal{R}^{+}(Y)$ and $\mathcal{R}(Y)$ instead of $\mathcal{R}^{I}\left(Y_{0}, \ldots, Y_{h-1}\right), \mathcal{R}^{+}\left(Y_{0}, \ldots, Y_{h-1}\right)$ and $\mathcal{R}\left(Y_{0}, \ldots, Y_{h-1}\right)$.

The rings $\mathcal{R}^{I}\left(\left\{Y_{j}\right\}_{j \in J}\right)$ are endowed with an $F$-linear action of $\Gamma_{F}$, given by the formula $a\left(Y_{j}\right)=\left[\sigma^{j}(a)\right]\left(Y_{j}\right)$. There is also an $F$-linear Frobenius map:

$$
\varphi_{q}: \mathcal{R}^{I}\left(\left\{Y_{j}\right\}_{j \in J}\right) \rightarrow \mathcal{R}^{I^{\prime}}\left(\left\{Y_{j}\right\}_{j \in J}\right),
$$

given by $Y_{j} \mapsto[p]\left(Y_{j}\right)$, for appropriate $I$ and $I^{\prime}$.

On the ring $\mathcal{R}^{I}(Y)$, we can define in addition an absolute $\sigma$-semilinear Frobenius map $\varphi$ by $Y_{j} \mapsto Y_{j+1}$ for $0 \leqslant j \leqslant h-2$ and $Y_{h-1} \mapsto[p]\left(Y_{0}\right)$. This map $\varphi$ has the property that $\varphi^{h}=\varphi_{q}$, and it also commutes with $\Gamma_{F}$.

Let $t_{i}=\log _{\mathrm{LT}}\left(Y_{i}\right)$. Since $a\left(Y_{i}\right)=\left[\sigma^{i}(a)\right]\left(Y_{i}\right)$ if $a \in \Gamma_{F}$, we have $a\left(t_{i}\right)=\sigma^{i}(a) \cdot t_{i}$ so that $g\left(t_{0} \ldots t_{h-1}\right)=\mathrm{N}_{F / \mathbf{Q}_{p}}\left(\chi_{\mathrm{LT}}(g)\right) \cdot t_{0} \ldots t_{h-1}=\chi_{\text {cyc }}(g) \cdot t_{0} \ldots t_{h-1}$ if $g \in G_{F}$ as well as $\varphi\left(t_{0} \ldots t_{h-1}\right)=p \cdot t_{0} \ldots t_{h-1}$. The element $t_{0} \ldots t_{h-1}$ therefore behaves like a $\mathbf{Q}_{p}$-multiple of the "usual" $t$ of $p$-adic Hodge theory (see Proposition 3.4 for a more precise statement).

The following two propositions are variations on the "Weierstrass division theorem."

Proposition 2.1. Let $I=[0 ; s]$ or $\left[0 ; s\left[\right.\right.$ and let $P(T) \in \mathcal{O}_{F}[T]$ be a monic polynomial of degree $d$ whose non-leading coefficients are all divisible by $p$. If $f \in \mathcal{R}^{I}\left(\left\{Y_{j}\right\}_{j \in J}\right)$, then there exists $g \in \mathcal{R}^{I}\left(\left\{Y_{j}\right\}_{j \in J}\right)$ and $f_{0}, \ldots, f_{d-1} \in \mathcal{R}^{I}\left(\left\{Y_{j}\right\}_{j \in J \backslash\{i\}}\right)$ such that

$$
f=f_{0}+f_{1} Y_{i}+\cdots+f_{d-1} Y_{i}^{d-1}+g \cdot P\left(Y_{i}\right) .
$$

Proof. If $I=[0 ; s]$ is closed, then this is a straightforward consequence of the Weierstrass division theorem. Since $g$ and the $f_{i}$ 's are uniquely determined, the result extends to the case when $I=[0 ; s[$.

Proposition 2.2. Let $I=[s ; s]$ and let $P(T) \in \mathcal{O}_{F}[T]$ be a monic polynomial of degree $d$, all of whose roots are of valuation $-1 / s$. If $f \in \mathcal{R}^{I}\left(\left\{Y_{j}\right\}_{j \in J}\right)$, then there exists $g \in \mathcal{R}^{I}\left(\left\{Y_{j}\right\}_{j \in J}\right)$ and $f_{0}, \ldots, f_{d-1} \in \mathcal{R}^{I}\left(\left\{Y_{j}\right\}_{j \in J \backslash\{i\}}\right)$ such that

$$
f=f_{0}+f_{1} Y_{i}+\cdots+f_{d-1} Y_{i}^{d-1}+g \cdot P\left(Y_{i}\right) .
$$

Proof. The polynomial $Q(T)=P(1 / T) T^{d} / P(0)$ is monic and all its roots are of valuation $1 / s$. Write $f=f^{+}+f^{-}$where $f^{+}$contains positive powers of $Y_{i}$ and $f^{-}$contains negative powers of $Y_{i}$. One may Weierstrass divide $f^{+}$by $P\left(Y_{i}\right)$ and $f^{-}$by $Q\left(1 / Y_{i}\right)$, which implies the proposition. 
Lemma 2.3. If $I$ is a closed interval, then the action of $\Gamma_{F}$ on $\mathcal{R}^{I}(Y)$ is locally $\mathbf{Q}_{p}$-analytic, and we have

$$
[1+a](f(Y))=f(Y)+\sum_{j=0}^{h-1} \sigma^{j}(a) \cdot \log _{\mathrm{LT}}\left(Y_{j}\right) \cdot \frac{d S}{d U}\left(Y_{j}, 0\right) \cdot \frac{d f}{d Y_{j}}(Y)+\mathrm{O}\left(a^{2}\right) .
$$

Proof. The above formula follows from the fact that $[1+a]\left(Y_{j}\right)=Y_{j} \oplus[a]\left(Y_{j}\right)=$ $Y_{j} \oplus\left(\sigma^{j}(a) \cdot \log _{\mathrm{LT}}\left(Y_{j}\right)+\mathrm{O}\left(a^{2}\right)\right)$.

Proposition 2.4. Let $\rho=\left(\rho_{1}, \ldots, \rho_{h-1}\right)$ and let $\mathcal{R}_{F_{k}}^{\rho}\left(T_{1}, \ldots, T_{h-1}\right)$ denote the ring of Laurent series converging for $\left|T_{i}\right|=\rho_{i}$, with coefficients in $F_{k}$. If the $z_{i} \in \mathfrak{m}_{\widehat{F}_{\infty}}$ are such that $\log _{\mathrm{LT}}\left(z_{i}\right) \neq 0,\left|z_{i}\right|=\rho_{i}$ and $g\left(z_{i}\right)=\left[\sigma^{i}(g)\right]\left(z_{i}\right)$ for $g \in \mathcal{O}_{F}^{\times}$, then the map $\mathcal{R}_{F_{k}}^{\rho}\left(T_{1}, \ldots, T_{h-1}\right) \rightarrow \mathbf{C}_{p}$ given by evaluating at $\left(z_{1}, \ldots, z_{h-1}\right)$ is injective.

Proof. Suppose that $f\left(z_{1}, \ldots, z_{h-1}\right)=0$ for some $f \in \mathcal{R}_{F_{k}}^{\rho}\left(T_{1}, \ldots, T_{h-1}\right)$. If $g \in \Gamma_{F_{k}}$, then $f\left(g\left(z_{1}\right), \ldots, g\left(z_{h-1}\right)\right)=0$. If $g=1+a$ with $a$ small, then Lemma 1.2 provides us with $h-1$ elements $y_{1}, \ldots, y_{h-1}$ of $\widehat{F}_{\infty}$ such that $g\left(z_{i}\right)=z_{i}+\sigma^{i}(a) \cdot y_{i}+\mathrm{O}\left(a^{2}\right)$. Since $y_{i}=\log _{\mathrm{LT}}\left(z_{i}\right) \cdot d S / d U\left(z_{i}, 0\right)$ and $d S / d U$ is a unit and $\log _{\mathrm{LT}}\left(z_{i}\right) \neq 0$, the elements $y_{1}, \ldots, y_{h-1}$ are all non-zero.

If $f \neq 0$ and $m$ is the smallest index for which $f$ has a non-zero partial derivative of order $m$ at $\left(z_{1}, \ldots, z_{h-1}\right)$ and if we expand $f\left(g\left(z_{1}\right), \ldots, g\left(z_{h-1}\right)\right)$ around $\left(z_{1}, \ldots, z_{h-1}\right)$ (which generalizes Lemma 2.3), then we get

$$
\begin{gathered}
\sum_{j_{1}+\cdots+j_{h-1}=m}\left(\sigma^{1}(a) y_{1}\right)^{j_{1}} \cdots\left(\sigma^{h-1}(a) y_{h-1}\right)^{j_{h-1}} \\
\times \frac{d^{m} f}{d T_{1}^{j_{1}} \cdots d T_{h-1}^{j_{h-1}}}\left(z_{1}, \ldots, z_{h-1}\right)+\mathrm{O}\left(a^{m+1}\right) .
\end{gathered}
$$

Since $f\left(g\left(z_{1}\right), \ldots, g\left(z_{h-1}\right)\right)=0$, the above linear combination is a homogeneous polynomial, of degree $m$ in $h-1$ variables and coefficients in $\widehat{F}_{\infty}$, that is identically zero on $\left(\sigma^{1}(a), \ldots, \sigma^{h-1}(a)\right)$. The shortest non-zero polynomial that is identically zero on $\left(\sigma^{1}(a), \ldots, \sigma^{h-1}(a)\right)$ can be taken to have coefficients in $F$ and Artin's theorem on the algebraic independence of characters implies that it is equal to zero. Since all the $y_{i}$ 's are non-zero, all the partial derivatives of order $m$ of $f$ are zero, so that finally $f=0$.

\section{Embeddings in $\mathbf{B}_{\mathrm{dR}}$}

We now explain how to embed the rings of power series of the previous section in the usual rings of $p$-adic periods. Let $\widetilde{\mathbf{B}}^{I}$ be the ring defined in Section 2.1 of [2]. This ring is complete with respect to the valuation $V(\cdot, I)$ (an equivalent valuation is denoted by $V_{I}(\cdot)$ in Section 2.1 of ibid.). Recall that if $x=\sum_{k \geqslant 0} p^{k}\left[x_{k}\right] \in \widetilde{\mathbf{A}}^{+}$, then $V(x, r)=\inf _{k}\left(\operatorname{val}_{\mathbf{E}}\left(x_{k}\right)+k r p /(p-1)\right)$. Set $r_{F}=p^{h-1} \cdot q /(q-1) \cdot(p-1) / p$ (for example, $r_{\mathbf{Q}_{p}}=1$ and if $h>1$, then $\left.r_{F}<p^{h-1}\right)$.

Proposition 3.1. If $r \geqslant r_{F}$ and $m \in \mathbf{Z}$, then $V\left(\varphi^{j}(u)^{m}, r\right)=m \cdot p^{j} \cdot q /(q-1)$ for $0 \leqslant j \leqslant h-1$. 
Proof. Recall that $u=\{\pi\}$ where $\pi=\left(\pi_{0}, \pi_{1}, \ldots\right)$ with $\operatorname{val}_{p}\left(\pi_{n}\right)=1 / q^{n-1}(q-1)$ for $n \geqslant 1$, so that $\operatorname{val}_{\mathbf{E}}(\pi)=q /(q-1)$. We have $\varphi^{j}(u)=\left[\pi^{p^{j}}\right]+\sum_{k \geqslant 1} p^{k}\left[u_{k, j}\right]$ where $\operatorname{val}_{\mathbf{E}}\left(u_{k, j}\right)>0$, so that if $r \geqslant r_{F}$, then $\varphi^{j}(u) /\left[\pi^{p^{j}}\right]$ is a unit of $\widetilde{\mathbf{A}}^{\dagger, r}$ and the proposition follows.

Note that a better estimate on the $\operatorname{val}_{\mathbf{E}}\left(u_{k, j}\right)$ would allow us to take a smaller value for $r_{F}$. Let $s_{n}=p^{n-h}(q-1)$ and let $r_{n}=p^{n-1}(p-1)$ (so that $s_{n} \cdot q /(q-1)=$ $\left.r_{n} \cdot p /(p-1)\right)$.

Proposition 3.2. If $n \geqslant h$, and if $f(Y) \in \mathcal{R}^{\left[s_{n} ; s_{n}\right]}(Y)$, then $f\left(u, \ldots, \varphi^{h-1}(u)\right)$ converges in $\widetilde{\mathbf{B}}^{\left[r_{n} ; r_{n}\right]}$.

Proof. If $f(Y)=\sum_{m \in \mathbf{Z}^{h}} a_{m} Y^{m} \in \mathcal{R}^{\left[s_{n} ; s_{n}\right]}(Y)$, then $\operatorname{val}_{p}\left(a_{m}\right)+w(m) /\left(p^{n-h}(q-1)\right) \rightarrow$ $+\infty$. If $n \geqslant h$, then $r_{n}>r_{F}$ so that $V\left(\varphi^{j}(u)^{m_{j}}, r\right)=m_{j} \cdot p^{j} \cdot q /(q-1)$ for $0 \leqslant j \leqslant h-1$ by Proposition 3.1, and then

$$
V\left(a_{m_{0}, \ldots, m_{h-1}} u^{m_{0}} \cdots \varphi^{h-1}(u)^{m_{h-1}}, r_{n}\right) \rightarrow+\infty .
$$

The series $f\left(u, \ldots, \varphi^{h-1}(u)\right)$ therefore converges in $\widetilde{\mathbf{B}}^{\left[r_{n} ; r_{n}\right]}$.

Corollary 3.3. If $n \geqslant h$, and if $f(Y) \in \mathcal{R}^{\left[0 ; s_{n}\right]}(Y)$, then $f\left(u, \ldots, \varphi^{h-1}(u)\right)$ converges in $\widetilde{\mathbf{B}}^{\left[0 ; r_{n}\right]}$. If $f(Y) \in \mathcal{R}^{+}(Y)$, then $f\left(u, \ldots, \varphi^{h-1}(u)\right)$ converges in $\widetilde{\mathbf{B}}_{\text {rig }}^{+}$.

Proof. If $f \in \mathcal{R}^{\left[0 ; s_{n}\right]}(Y)$, then each term of the series $f\left(u, \ldots, \varphi^{h-1}(u)\right)$ belongs to $\widetilde{\mathbf{B}}^{+}$so that it converges in $\widetilde{\mathbf{B}}^{\left[0 ; r_{n}\right]}$ by the maximum modulus principle (corollary 2.20 of [2]). The second assertion follows by passing to the limit.

The image of $\log _{\mathrm{LT}}\left(Y_{0}\right) \cdots \log _{\mathrm{LT}}\left(Y_{h-1}\right)$ in $\widetilde{\mathbf{B}}_{\text {rig }}^{+} \subset \mathbf{B}_{\mathrm{dR}}^{+}$is $a \cdot t$ with $a \in \mathbf{Q}_{p}$, as we have seen above. We henceforth denote by $t$ the element of $\mathcal{R}^{+}(Y)$ whose image in $\widetilde{\mathbf{B}}_{\text {rig }}^{+}$is $t$, that is $t=\log _{\mathrm{LT}}\left(Y_{0}\right) \cdots \log _{\mathrm{LT}}\left(Y_{h-1}\right) / a$. In the following proposition, we determine the valuation of $a$ (this is not used in the rest of this article).

Proposition 3.4. In the ring $\mathbf{B}_{\mathrm{dR}}^{+}$, the product $\log _{\mathrm{LT}}(u) \cdots \log _{\mathrm{LT}}\left(\varphi^{h-1}(u)\right)$ belongs to $p^{h-1} \cdot \mathbf{Z}_{p}^{\times} \cdot t$, where $t$ is the usual $t$ of $p$-adic Hodge theory.

Proof. We have seen that $\log _{\mathrm{LT}}(u) \cdots \log _{\mathrm{LT}}\left(\varphi^{h-1}(u)\right)=a \cdot t$ with $a \in \mathbf{Q}_{p}$, and we now compute $\operatorname{val}_{p}(a)$. We have $\log _{\mathrm{LT}}(u)=u \cdot \prod_{k \geqslant 1} Q_{k}(u) / p$ and likewise, if $\pi=[\varepsilon]-1$, then $t=\pi \cdot \prod_{k \geqslant 1} Q_{k}^{\text {cyc }}(\pi) / p$. This implies that $\theta\left(t / \log _{\mathrm{LT}}(u)\right)=\theta(\pi / u)$. Since both $\pi / \varphi^{-1}(\pi)$ and $u / \varphi_{q}^{-1}(u)$ are generators of $\operatorname{ker}(\theta)$ in $\widetilde{\mathbf{A}}^{+}$, we have $\operatorname{val}_{p}\left(\theta\left(t / \log _{\mathrm{LT}}(u)\right)\right)=$ $1 /(p-1)-1 /(q-1)$. On the other hand, $\operatorname{val}_{p}\left(\theta \circ \varphi^{j}(u)\right)=\operatorname{val}_{p}\left(\lim _{n \rightarrow \infty}\left[p^{n}\right]\left(\pi_{n}^{p^{j}}\right)\right)=$ $1+p^{j} /(q-1)$ if $1 \leqslant j \leqslant h-1$, so that $\operatorname{val}_{p}\left(\theta\left(\log _{\mathrm{LT}}\left(\varphi^{j}(u)\right)\right)\right)=1+p^{j} /(q-1)$. This implies that $\operatorname{val}_{p}(a)=\operatorname{val}_{p}(\theta(a))=h-1$, and hence the proposition.

Definition 3.5. Let $\iota_{n}: \mathcal{R}^{\left[s_{n} ; s_{n}\right]}(Y) \rightarrow \mathbf{B}_{\mathrm{dR}}^{+}$be the compositum of the map defined above, with the map $\varphi^{-n}: \widetilde{\mathbf{B}}^{\left[r_{n} ; r_{n}\right]} \rightarrow \widetilde{\mathbf{B}}^{\left[r_{0} ; r_{0}\right]}$ and the map $\widetilde{\mathbf{B}}^{\left[r_{0} ; r_{0}\right]} \subset \mathbf{B}_{\mathrm{dR}}^{+}$defined in Section 2.2 of [2].

It follows from the definition as well as the formulas for $\varphi$ and the action of $\Gamma_{F}$ on $\mathcal{R}^{I}(Y)$ that $\iota_{n+1}(\varphi(f))=\iota_{n}(f)$ when applicable, and that $g\left(\iota_{n}(f)\right)=\iota_{n}(g(f))$ if $g \in G_{F}$. Since $\iota_{n}(t)=p^{-n} t$, we can extend $\iota_{n}$ to $\iota_{n}: \mathcal{R}^{\left[s_{n} ; s_{n}\right]}(Y)[1 / t] \rightarrow \mathbf{B}_{\mathrm{dR}}$. 
Theorem 3.6. If $n \geqslant h$, if $f \in \mathcal{R}^{\left[s_{n} ; s_{n}\right]}(Y)$, and if $n=h k+i$ with $0 \leqslant i \leqslant h-1$, then we have $\iota_{n}(f) \in \mathrm{Fil}^{1} \mathbf{B}_{\mathrm{dR}}^{+}$if and only if $f \in Q_{k}\left(Y_{i}\right) \cdot \mathcal{R}^{\left[s_{n} ; s_{n}\right]}(Y)$.

Proof. Recall that $u=\left\{\left(\pi_{0}, \pi_{1}, \ldots\right)\right\} \in \widetilde{\mathbf{A}}^{+}$. If $m \geqslant 1$ and $u_{m}=\theta\left(\varphi^{-m}(u)\right) \in \widehat{F}_{\infty}$, then $g\left(u_{m}\right)=\left[\sigma^{-m}(g)\right]\left(u_{m}\right)$. Note that if $m=h \ell$, then $u_{m}=\theta\left(\varphi_{q}^{-\ell}(u)\right)=\pi_{\ell}$. The theorem is equivalent to the assertion that $f^{\sigma^{-n}}\left(u_{n}, \ldots, u_{n-h+1}\right)=0$ in $\mathbf{C}_{p}$ if and only if $f \in Q_{k}\left(Y_{i}\right) \cdot \mathcal{R}^{\left[s_{n} ; s_{n}\right]}(Y)$. We have $u_{n-i}=\pi_{k}$ so that if $f$ belongs to $Q_{k}\left(Y_{i}\right) \cdot \mathcal{R}^{\left[s_{n} ; s_{n}\right]}(Y)$, then $f^{\sigma^{-n}}\left(u_{n}, \ldots, u_{n-h+1}\right)=0$.

Since $Q_{k}(T)$ is a monic polynomial of degree $d=q^{k-1}(q-1)$, whose non-leading coefficients are divisible by $p$, we can use Proposition 2.2 to write $f^{\sigma^{-n}}=f_{0}+Y_{i} f_{1}+$ $\cdots+Y_{i}^{d-1} f_{d-1}+Q_{k}\left(Y_{i}\right) r$ with $f_{i}$ a power series in the $Y_{j}$ 's with $j \neq i$. Proposition 2.4 applied to $f_{0}+\pi_{k} f_{1}+\cdots+\pi_{k}^{d-1} f_{d-1}$, with the $T_{j}$ 's a suitable permutation of the $Y_{j}$ 's, shows that $f_{0}+\pi_{k} f_{1}+\cdots+\pi_{k}^{d-1} f_{d-1}=0$. Therefore, $f=Q_{k}\left(Y_{i}\right) r^{\sigma^{n}}$, which proves the theorem.

Corollary 3.7. If $n \geqslant h$, then the map $\iota_{n}: \mathcal{R}^{\left[s_{n} ; s_{n}\right]}(Y) \rightarrow \mathbf{B}_{\mathrm{dR}}^{+}$is injective. If $n \in \mathbf{Z}$, then the map $\iota_{n}: \mathcal{R}^{+}(Y) \rightarrow \mathbf{B}_{\mathrm{dR}}^{+}$is injective.

Proof. The first assertion follows from Theorem 3.6. The second follows from that, and from the fact that $\iota_{n+1}(\varphi(f))=\iota_{n}(f)$ for the other $n$.

Corollary 3.8. If $I \subset\left[s_{h} ;+\infty\left[\right.\right.$, and if $f(Y) \in \mathcal{R}^{I}(Y)[1 / t]$, then $f(Y) \in \mathcal{R}^{I}(Y)$ if and only if $\iota_{n}(f) \in \mathbf{B}_{\mathrm{dR}}^{+}$for all $n$ such that $s_{n} \in I$.

\section{4. $\left(\varphi_{q}, \Gamma_{F}\right)$-modules in one variable}

Before constructing $\left(\varphi_{q}, \Gamma_{F}\right)$-modules over $\mathcal{R}(Y)$, we review Kisin and Ren's construction of $\left(\varphi_{q}, \Gamma_{F}\right)$-modules in one variable and explain why we need rings in several variables.

Let $Y_{0}$ be the variable of Section 2 , and let $\mathcal{E}\left(Y_{0}\right)$ be Fontaine's field of [12] with coefficients in $F$, that is $\mathcal{E}\left(Y_{0}\right)=\mathcal{O}_{\mathcal{E}}\left(Y_{0}\right)[1 / p]$ where $\mathcal{O}_{\mathcal{E}}\left(Y_{0}\right)$ is the $p$-adic completion of $\mathcal{O}_{F} \llbracket Y_{0} \rrbracket\left[1 / Y_{0}\right]$. We let $\mathcal{E}^{\dagger}\left(Y_{0}\right)$ and $\mathcal{R}\left(Y_{0}\right)$ denote the corresponding overconvergent and Robba rings. If $I$ is a subinterval of $[0 ;+\infty]$, then we denote as above by $\mathcal{R}^{I}\left(Y_{0}\right)$ the set of power series $f\left(Y_{0}\right)=\sum_{m \in \mathbf{Z}} a_{m} Y_{0}^{m}$ that belong to $\mathcal{R}^{I}\left(Y_{0}, \ldots, Y_{h-1}\right)$ via the natural inclusion.

If $K / F$ is a finite extension, then by the theory of the field of norms (see [14,22]), there corresponds to it a finite extension $\mathcal{E}_{K}\left(Y_{0}\right)$ of $\mathcal{E}\left(Y_{0}\right)$, of degree $\left[K_{\infty}: F_{\infty}\right]$. A $\left(\varphi_{q}, \Gamma_{K}\right)$-module over $\mathcal{E}_{K}\left(Y_{0}\right)$ is a finite dimensional $\mathcal{E}_{K}\left(Y_{0}\right)$-vector space $\mathrm{D}$, along with a semilinear $\varphi_{q}$ and a compatible action of $\Gamma_{K}$. We say that $\mathrm{D}$ is étale if $\mathrm{D}=\mathcal{E}_{K}\left(Y_{0}\right) \otimes_{\mathcal{O}_{\mathcal{E}_{K}}\left(Y_{0}\right)} \mathrm{D}_{0}$ where $\mathrm{D}_{0}$ is a $\left(\varphi_{q}, \Gamma_{K}\right)$-module over $\mathcal{O}_{\mathcal{E}_{K}}\left(Y_{0}\right)$. By specializing the constructions of [12], Kisin and Ren prove the following theorem in their paper (Theorem 1.6 of [18]).

Theorem 4.1. The functors

$$
V \mapsto\left(\widehat{\mathcal{E}}\left(Y_{0}\right)^{\mathrm{unr}} \otimes_{F} V\right)^{H_{K}} \text { and } \mathrm{D} \mapsto\left(\widehat{\mathcal{E}}\left(Y_{0}\right)^{\mathrm{unr}} \otimes_{\mathcal{E}_{K}\left(Y_{0}\right)} \mathrm{D}\right)^{\varphi_{q}=1}
$$

give rise to mutually inverse equivalences of categories between the category of $F$-linear representations of $G_{K}$ and the category of étale $\left(\varphi_{q}, \Gamma_{K}\right)$-modules over $\mathcal{E}_{K}\left(Y_{0}\right)$. 
We say that an $F$-linear representation of $G_{K}$ is $F$-analytic if it is Hodge-Tate with weights 0 (i.e., $\mathbf{C}_{p}$-admissible) at all embeddings $\tau \neq \mathrm{Id}$. Kisin and Ren then go on to show that if $K \subset F_{\infty}$, and if $V$ is a crystalline representation of $G_{K}$, that is $F$-analytic, then the $\left(\varphi_{q}, \Gamma_{K}\right)$-module attached to $V$ is overconvergent (see Section 3.3 of ibid.).

Assume from now on that $K \subset F_{\infty}$, so that $\mathcal{E}_{K}\left(Y_{0}\right)=\mathcal{E}\left(Y_{0}\right)$. If $\mathrm{D}$ is a $\left(\varphi_{q}, \Gamma_{K}\right)$ module over $\mathcal{R}\left(Y_{0}\right)$, and if $g \in \Gamma_{K}$ is close enough to 1 , then by standard arguments (see Section 4.1 of [2] or Section 2.1 of [18]), the series $\log (g)=\log (1+(g-1)$ ) gives rise to a differential operator $\nabla_{g}: \mathrm{D} \rightarrow \mathrm{D}$. The map Lie $\Gamma_{F} \rightarrow \operatorname{End}(\mathrm{D})$ arising from $v \mapsto \nabla_{\exp (v)}$ is $\mathbf{Q}_{p}$-linear, and we say that $\mathrm{D}$ is $F$-analytic if this map is $F$-linear (see Section 2.1 of [18] and Section 1.3 of [15]). This is equivalent to the requirement that $\nabla_{j}=0$ on $\mathrm{D}$ for $1 \leqslant j \leqslant h-1$, where $\nabla_{j}$ is the partial derivative in the direction $\sigma^{j}$.

Theorem 4.2. If $V$ is an overconvergent $F$-linear representation of $G_{K}$, and if $\mathrm{D}(V)=\mathcal{R}\left(Y_{0}\right) \otimes_{\mathcal{E}^{\dagger}\left(Y_{0}\right)} \mathrm{D}^{\dagger}(V)$, then $\mathrm{D}(V)$ is $F$-analytic if and only if $V$ is $F$-analytic.

Proof. Choose $1 \leqslant j \leqslant h-1$, and take $n \gg 0$ such that $n=-j \bmod h$. By Proposition 3.2 , we have a map $\theta \circ \varphi^{-n}: \mathcal{R}^{\left[s_{n} ; s_{n}\right]}\left(Y_{0}\right) \rightarrow \mathbf{B}_{\mathrm{dR}}^{+} \rightarrow \mathbf{C}_{p}$, giving rise to an isomorphism

$$
\mathbf{C}_{p} \otimes_{\mathcal{R}^{\left[s_{n} ; s_{n}\right]}\left(Y_{0}\right)}^{\theta \circ \varphi^{-n}} \mathrm{D}^{\left[s_{n} ; s_{n}\right]}(V) \rightarrow \mathbf{C}_{p} \otimes_{F}^{\sigma^{j}} V
$$

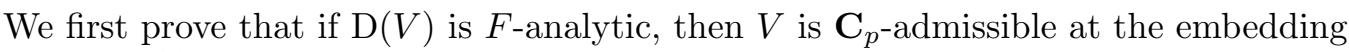
$\sigma^{j}$. Let $\widehat{F}_{\infty}^{(j)}$ denote the field of locally $\sigma^{j}$-analytic vectors of $\widehat{F}_{\infty}$ for the action of $\Gamma_{K}$. Note that $\theta \circ \varphi^{-n}\left(\mathcal{R}^{\left[s_{n} ; s_{n}\right]}\left(Y_{0}\right)\right) \subset \widehat{F}_{\infty}^{(j)}$. Let $\mathrm{D}_{\text {Sen }}^{(j)}(V)$ be the $\widehat{F}_{\infty}^{(j)}$-vector space

$$
\mathrm{D}_{\text {Sen }}^{(j)}(V)=\widehat{F}_{\infty}^{(j)} \otimes_{\theta \circ \varphi^{-n}\left(\mathcal{R}^{\left[s_{n} ; s_{n}\right]}\left(Y_{0}\right)\right)} \theta \circ \varphi^{-n}\left(\mathrm{D}^{\left[s_{n} ; s_{n}\right]}(V)\right) .
$$

It is of dimension $d$, its image in $\left(\mathbf{C}_{p} \otimes_{F}^{\sigma^{j}} V\right)^{H_{F}}$ generates $\mathbf{C}_{p} \otimes_{F}^{\sigma^{j}} V$, and its elements are all locally $\sigma^{j}$-analytic vectors of $\left(\mathbf{C}_{p} \otimes_{F}^{\sigma^{j}} V\right)^{H_{F}}$ because $\mathrm{D}(V)$ is $F$-analytic and $\varphi^{-n} \circ \nabla_{j}=\nabla_{0} \circ \varphi^{-n}$. If $y \in \mathrm{D}_{\text {Sen }}^{(j)}(V)$, then $(g(y)-y) /\left(\sigma^{j} \circ \chi_{\mathrm{LT}}(g)-1\right)$ has a limit as $g \rightarrow 1$, and we call $\nabla_{j}(y)$ this limit. We then have $g(y)=\exp \left(\log _{p}\left(\sigma^{j} \circ \chi_{\mathrm{LT}}(g)\right) \cdot \nabla_{j}\right)(y)$ if $g \in \Gamma_{K}$ is close to 1 .

Recall that there exists $a_{j} \in \mathbf{C}_{p}$ such that $\log _{p}\left(\sigma^{j} \circ \chi_{\mathrm{LT}}(g)\right)=g\left(a_{j}\right)-a_{j}$. For example, one can take $a_{j}=\log _{p}\left(\theta \circ \iota_{0}\left(t_{j}\right)\right)$. The element $a_{j}$ then belongs to $\widehat{F}_{\infty}^{(j)}$ for obvious reasons and satisfies $\nabla_{j}\left(a_{j}\right)=1$. Take $y \in \mathrm{D}_{\text {Sen }}^{(j)}(V)$, and choose $a_{j, 0} \in F_{\infty}$ such that $\left|a_{j}-a_{j, 0}\right|_{p}$ is small enough. The series

$$
C(y)=\sum_{k \geqslant 0}(-1)^{k} \frac{\left(a_{j}-a_{j, 0}\right)^{k}}{k !} \nabla_{j}^{k}(y)
$$

then converges for the topology of $\mathrm{D}_{\text {Sen }}^{(j)}(V)$ (the technical details concerning convergence in such spaces of locally analytic vectors can be found in [6]) and a short computation shows that $\nabla_{j}(C(y))=0$, so that $C(y) \in\left(\mathbf{C}_{p} \otimes_{F}^{\sigma^{j}} V\right)^{G_{F_{n}}}$ for some $n=n(y) \gg 0$. In addition, $n(y)=n\left(\nabla_{j}^{k}(y)\right)$ for $k \geqslant 0$, the series for $C\left(\nabla_{j}^{k}(y)\right)$ also converges for the topology of $\mathrm{D}_{\text {Sen }}^{(j)}(V)$, and $y=\sum_{k \geqslant 0}\left(a_{j}-a_{j, 0}\right)^{k} / k ! \cdot C\left(\nabla_{j}^{k}(y)\right)$.

If $y_{1}, \ldots, y_{d}$ is a basis of $\mathrm{D}_{\text {Sen }}^{(j)}(V)$, and if $n \geqslant \max n\left(y_{i}\right)$, then the above computations show that the elements $y_{i}$ belong to $\widehat{F}_{\infty}^{(j)} \otimes_{F_{n}}\left(\mathbf{C}_{p} \otimes_{F}^{\sigma^{j}} V\right)^{G_{F_{n}}}$, so that 
$\left(\mathbf{C}_{p} \otimes_{F}^{\sigma^{j}} V\right)^{G_{F_{n}}}$ generates $\left(\mathbf{C}_{p} \otimes_{F}^{\sigma^{j}} V\right)^{H_{F}}$. This implies that $V$ is $\mathbf{C}_{p^{-}}$-admissible at the embedding $\sigma^{j}$. This is true for all $1 \leqslant j \leqslant h-1$, and therefore $V$ is $F$-analytic.

We now prove that if $V$ is $\mathbf{C}_{p}$-admissible at the embedding $\sigma^{j}$, then $\nabla_{j}=0$ on $\mathrm{D}(V)$. Choose $n=h m-j$ with $m \gg 0$. Since $j \neq 0 \bmod h$, the $\operatorname{map} \theta \circ \varphi^{-n}$ : $\mathcal{R}^{\left[s_{n} ; s_{n}\right]}\left(Y_{0}\right) \rightarrow \mathbf{C}_{p}$ is injective by Theorem 3.6. This implies that the map

$$
\mathrm{D}^{\left[s_{n} ; s_{n}\right]}(V) \rightarrow \mathbf{C}_{p} \otimes_{\mathcal{R}^{\left[s_{n} ; s_{n}\right]}\left(Y_{0}\right)}^{\theta \circ \varphi^{-n}} \mathrm{D}^{\left[s_{n} ; s_{n}\right]}(V)
$$

is injective, and hence the map $\mathrm{D}^{\left[s_{n} ; s_{n}\right]}(V) \rightarrow \mathbf{C}_{p} \otimes_{F}^{\sigma^{j}} V$ is also injective. Therefore, we have an injection $\mathrm{D}^{\left[s_{n} ; s_{n}\right]}(V) \rightarrow\left(\left(\mathbf{C}_{p} \otimes_{F}^{\sigma^{j}} V\right)^{H_{F}}\right)^{\text {an }}$ where $\left(\left(\mathbf{C}_{p} \otimes_{F}^{\sigma^{j}} V\right)^{H_{F}}\right)^{\text {an }}$ denotes the set of locally $\mathbf{Q}_{p}$-analytic vectors of $\left(\mathbf{C}_{p} \otimes_{F}^{\sigma^{j}} V\right)^{H_{F}}$. If $V$ is $\mathbf{C}_{p}$-admissible at the embedding $\sigma^{j}$, then $\left(\left(\mathbf{C}_{p} \otimes \sigma_{F}^{j} V\right)^{H_{F}}\right)^{\text {an }}=\left(\widehat{F}_{\infty}^{\text {an }}\right)^{d}$. One of the main results of [6] is that $\nabla_{0}=0$ on $\widehat{F}_{\infty}^{\text {an }}$ (it is shown in [6] that, in a suitable sense, $\widehat{F}_{\infty}^{\text {an }}$ is generated by $F_{\infty}$ and the elements $\left.a_{1}, \ldots, a_{h-1}\right)$. This implies that $\nabla_{j}=0$ on $\mathrm{D}^{\left[s_{n} ; s_{n}\right]}(V)$, since $\varphi^{-n} \circ \nabla_{j}=\nabla_{0} \circ \varphi^{-n}$.

Note that an analogous argument for the proof of the implication " $\mathrm{D}(V)$ is $F$-analytic implies $V$ is $F$-analytic" was given by Bingyong Xie for those $V$ that are trivial on $H_{F}$.

Corollary 4.3. If $V$ is an absolutely irreducible $F$-linear overconvergent representation of $G_{K}$, then there exists a character $\delta$ of $\Gamma_{K}$ such that $V \otimes \delta$ is F-analytic.

Proof. We give a sketch of the proof. Choose some $g \in \Gamma_{K}$ such that $\log _{p}\left(\chi_{\mathrm{LT}}(g)\right) \neq 0$, and let $\nabla=\log (g) / \log _{p}\left(\chi_{\mathrm{LT}}(g)\right)$. Choose $r>0$ large enough and $s \geqslant q r$. If $a \in \mathcal{O}_{F}$, and if $\operatorname{val}_{p}(a) \geqslant n$ for $n=n(r, s)$ large enough, then the series $\exp (a \cdot \nabla)$ converges to an operator on $\mathrm{D}^{[r ; s]}(V)$. This way, we can define a twisted action of $\Gamma_{K_{n}}$ on $\mathrm{D}^{[r ; s]}(V)$, by the formula $h \star x=\exp \left(\log _{p}\left(\chi_{\mathrm{LT}}(h)\right) \cdot \nabla\right)(x)$. This action is now $F$-analytic by construction.

Since $s \geqslant q r$, the modules $\mathrm{D}^{\left[q^{m} r ; q^{m} s\right]}(V)$ for $m \geqslant 0$ are glued together by $\varphi_{q}$ and this way, we get a new action of $\Gamma_{K_{n}}$ on $\mathrm{D}(V)$. Since $\varphi_{q}$ is unchanged, this new $\left(\varphi_{q}, \Gamma_{K_{n}}\right)$-module is étale, and therefore corresponds to a representation $W$ of $G_{K_{n}}$. This representation $W$ is $F$-analytic by Theorem 4.2 , and its restriction to $H_{F}$ is isomorphic to $V$.

The space $\operatorname{Hom}\left(V, \operatorname{ind}_{G_{K}}^{G_{K}} W\right)^{H_{F}}$ is non-empty, and is a finite dimensional representation of $\Gamma_{K}$. Since $\Gamma_{K}$ is abelian, we find (possibly extending scalars) a character $\delta$ of $\Gamma_{K}$ and a non-zero $f \in \operatorname{Hom}\left(V, \operatorname{ind}_{G_{K_{n}}}^{G_{K}} W\right)^{H_{F}}$ such that $h(f)=\delta(h) \cdot f$ if $h \in G_{K}$. This $f$ gives rise to a non-zero $G_{K}$-equivariant map $V \otimes \delta \rightarrow \operatorname{ind}_{G_{K_{n}}}^{G_{K}} W$. Since $\operatorname{ind}_{G_{K_{n}}}^{G_{K}} W$ is $F$-analytic and $V$ is absolutely irreducible, the corollary follows.

Corollary 4.3 (as well as Theorem 0.6 of [15]) suggests that if we want to attach overconvergent $\left(\varphi_{q}, \Gamma_{K}\right)$-modules to all $F$-linear representations of $G_{K}$, then we need to go beyond the objects in only one variable. We finish with a conjecture that seems reasonable enough, since it holds for crystalline representations by the work of Kisin and Ren (see also Theorem 0.3 of [15]).

Conjecture 4.4. If $V$ is $F$-analytic, then it is overconvergent. 


\section{Construction of $\mathcal{R}^{+}(\boldsymbol{Y})$-modules}

We now explain how to construct some $\mathcal{R}^{+}(Y)$-modules $\mathrm{M}^{+}(D)$ that are attached to some filtered $\varphi_{q}$-modules $D$. Let $D$ be a finite dimensional $F$-vector space, endowed with an $F$-linear Frobenius map $\varphi_{q}: D \rightarrow D$, and an action of $G_{F}$ on $D$ that factors through $\Gamma_{F}$ and commutes with $\varphi_{q}$.

For each $0 \leqslant j \leqslant h-1$, let $\mathrm{Fil}_{j}^{\bullet}$ be a filtration on $F \otimes_{F}^{\sigma^{j}} D \simeq D$ that is stable under $\Gamma_{F}$. If $n \in \mathbf{Z}$, let $\mathbf{B}_{\mathrm{dR}} \otimes_{F}^{\sigma^{n}} D$ denote the tensor product of $\mathbf{B}_{\mathrm{dR}}$ and $D$ above $F$, where $F$ maps to $\mathbf{B}_{\mathrm{dR}}$ via $\sigma^{n}$. We then have $b \otimes a \cdot d=\sigma^{n}(a) \cdot b \otimes d$. Note that $\mathbf{B}_{\mathrm{dR}} \otimes_{F}^{\sigma^{n}} D$ only depends on $n \bmod h$. Define $W_{\mathrm{dR}}^{+, j}(D)=\mathrm{Fil}_{j}^{0}\left(\mathbf{B}_{\mathrm{dR}} \otimes_{F}^{\sigma^{j}} D\right)$ so that $W_{\mathrm{dR}}^{+, j}$ is a $G_{F}$-stable $\mathbf{B}_{\mathrm{dR}}^{+}$-lattice of $\mathbf{B}_{\mathrm{dR}} \otimes_{F}^{\sigma^{j}} D$.

Example 5.1. If $V$ is an $F$-linear crystalline representation of $G_{F}$ of dimension $d$, then $\mathrm{D}_{\text {cris }}(V)$ is a free $F \otimes \mathbf{Q}_{p} F$-module of rank $d$ and we have

$$
\mathrm{D}_{\text {cris }}(V)=D \oplus \varphi(D) \oplus \cdots \oplus \varphi^{h-1}(D),
$$

according to the decomposition of $F \otimes \mathbf{Q}_{p} F$ as $\prod_{\sigma^{i}: F \rightarrow F} F$. Each $\varphi^{j}(D)$ comes with the filtration induced from $\mathrm{D}_{\text {cris }}(V)$, and we set $\operatorname{Fil}_{j}^{k} D=\varphi^{-j}\left(\operatorname{Fil}^{k} \mathrm{D}_{\text {cris }}(V) \cap \varphi^{j}(D)\right)$.

We now briefly recall some definitions from [21]. The ring $\mathcal{R}^{+}(Y)$ is a Fréchet-Stein algebra; indeed, its topology is defined by the valuations $\left\{W\left(\cdot,\left[0 ; s_{n}\right]\right)\right\}_{n \in S}$, where $S$ is any unbounded set of integers, and the ring $\mathcal{R}^{\left[0 ; s_{n}\right]}(Y)$ is noetherian and flat over $\mathcal{R}^{\left[0 ; s_{m}\right]}(Y)$ if $m \geqslant n \in S$. Recall that a coherent sheaf is then a family $\left\{M^{\left[0 ; s_{n}\right]}\right\}_{n \in S}$ of finitely generated $\mathcal{R}^{\left[0 ; s_{n}\right]}(Y)$-modules, such that $\mathcal{R}^{\left[0 ; s_{n}\right]}(Y) \otimes_{\mathcal{R}^{\left[0 ; s_{m}\right]}(Y)} M^{\left[0 ; s_{m}\right]}=$ $M^{\left[0 ; s_{n}\right]}$ for all $m \geqslant n \in S$. A $\mathcal{R}^{+}(Y)$-module $M$ is said to be coadmissible if $M$ is the set of global sections of a coherent sheaf $\left\{M^{\left[0 ; s_{n}\right]}\right\}_{n \in S}$. We say that $M$ is a reflexive coadmissible $\mathcal{R}^{+}(Y)$-module if each $M^{\left[0 ; s_{n}\right]}$ is a reflexive $\mathcal{R}^{\left[0 ; s_{n}\right]}(Y)$-module. By Lemma 8.4 of [21], this is the same as requiring that $M$ itself be a reflexive $\mathcal{R}^{+}(Y)$-module.

Let $\lambda_{j}=\log _{\mathrm{LT}}\left(Y_{j}\right) / Y_{j}$ and $\lambda=\lambda_{0} \cdots \lambda_{h-1}$, so that for any $n \in \mathbf{Z}, t$ is a $\mathbf{Q}_{p}$-multiple of $\iota_{n}\left(\lambda \cdot Y_{0} \cdots Y_{h-1}\right)$. Let $f_{j}=\lambda / \lambda_{j}$, so that if $k=j \bmod h$, then $\iota_{k}\left(f_{j}\right)$ is a unit of $\mathbf{B}_{\mathrm{dR}}^{+}$.

$$
\text { If } y=\sum_{i} y_{i} \otimes d_{i} \in \mathcal{R}^{+}(Y)[1 / \lambda] \otimes_{F} D \text {, let } \iota_{k}(y)=\sum_{i} \iota_{k}\left(y_{i}\right) \otimes d_{i} \in \mathbf{B}_{\mathrm{dR}} \otimes_{F}^{\sigma^{-k}} D \text {. }
$$

Definition 5.2. Let $\mathrm{M}^{+}(D)$ be the set of $y \in \mathcal{R}^{+}(Y)[1 / \lambda] \otimes_{F} D$ that satisfy $\iota_{k}(y) \in$ $W_{\mathrm{dR}}^{+,-k}(D)$ for all $k \geqslant h$.

Theorem 5.3. If $D$ is a $\varphi_{q}$-module with an action of $\Gamma_{F}$ and $h$ filtrations, then

(1) $\mathrm{M}^{+}(D)$ is a reflexive coadmissible $\mathcal{R}^{+}(Y)$-module;

(2) the $\mathcal{R}^{+}(Y)\left[1 / f_{j}\right]$-module $\mathrm{M}^{+}(D)\left[1 / f_{j}\right]$ is free of rank $d$ for $0 \leqslant j \leqslant h-1$;

(3) $\mathrm{M}^{+}(D)=\cap_{j=0}^{h-1} \mathrm{M}^{+}(D)\left[1 / f_{j}\right]$.

In the remainder of this section, we prove Theorem 5.3. We now establish some preliminary results. Let $S=\{h m+(h-1)$ where $m \geqslant 1\}$, and take $n \in S$. Recall that on the ring $\mathcal{R}^{\left[0 ; s_{n}\right]}(Y)$, the map $\iota_{k}$ is defined for $h \leqslant k \leqslant n$. Let

$$
\mathrm{M}(D)^{\left[0 ; s_{n}\right]}=\left\{y \in \mathcal{R}^{\left[0 ; s_{n}\right]}(Y)[1 / \lambda] \otimes_{F} D, \iota_{k}(y) \in W_{\mathrm{dR}}^{+,-k}(D) \text { for all } h \leqslant k \leqslant n\right\} .
$$


For $0 \leqslant j \leqslant h-1$, recall that $\mathcal{R}^{I}\left(Y_{j}\right)$ is a ring of power series in one variable. Let

$$
\begin{aligned}
N_{j}^{\left[0 ; s_{n}\right]} & =\left\{y \in \mathcal{R}^{\left[0 ; s_{n}\right]}\left(Y_{j}\right)\left[1 / \lambda_{j}\right] \otimes_{F} D, \iota_{k h+j}(y) \in W_{\mathrm{dR}}^{+,-j}(D) \text { for all } 1 \leqslant k \leqslant m\right\}, \\
N_{j}^{+} & =\left\{y \in \mathcal{R}^{+}\left(Y_{j}\right)\left[1 / \lambda_{j}\right] \otimes_{F} D, \iota_{k h+j}(y) \in W_{\mathrm{dR}}^{+,-j}(D) \text { for all } k \geqslant 1\right\} .
\end{aligned}
$$

Since $\mathcal{R}^{+}\left(Y_{j}\right)=\varphi^{j}\left(\mathcal{R}^{+}\left(Y_{0}\right)\right)$ if $0 \leqslant j \leqslant h-1$, the construction of $N_{j}^{+}$is completely analogous to that of $\mathcal{M}\left(F \otimes_{F}^{\sigma^{-j}} D\right)$, given for example in Section 2.2 of [18].

Proposition 5.4. The $\mathcal{R}^{+}\left(Y_{j}\right)$-module $N_{j}^{+}$is free of rank $d$, for all $n$ we have $N_{j}^{\left[0 ; s_{n}\right]}=\mathcal{R}^{\left[0 ; s_{n}\right]}\left(Y_{j}\right) \otimes_{\mathcal{R}^{+}\left(Y_{j}\right)} N_{j}^{+}$, and the map $\mathbf{B}_{\mathrm{dR}}^{+} \otimes_{\mathcal{R}^{+}\left(Y_{j}\right)}^{\iota_{k h+j}} N_{j}^{+} \rightarrow W_{\mathrm{dR}}^{+,-j}(D)$ is an isomorphism for all $k \geqslant 1$.

Proof. Since there is only one variable, the proof is a standard argument, analogous to the one which one can find in Section II.1 of [4] or Section 2.2 of [18].

Let $M_{j}^{\left[0 ; s_{n}\right]}=\mathcal{R}^{\left[0 ; s_{n}\right]}(Y)\left[1 / f_{j}\right] \otimes_{\mathcal{R}^{\left[0 ; s_{n}\right]}\left(Y_{j}\right)} N_{j}^{\left[0 ; s_{n}\right]}$, where $f_{j}=\lambda / \lambda_{j}$.

Proposition 5.5. We have $\mathrm{M}(D)^{\left[0 ; s_{n}\right]}\left[1 / f_{j}\right]=M_{j}^{\left[0 ; s_{n}\right]}$ and $\mathrm{M}(D)^{\left[0 ; s_{n}\right]}=\cap_{j} M_{j}^{\left[0 ; s_{n}\right]}$.

Proof. In the sequel, we use the fact that $Q_{1}\left(Y_{j}\right) \cdots Q_{m}\left(Y_{j}\right)$ and $\lambda_{j}$ generate the same ideal of $\mathcal{R}^{\left[0 ; s_{n}\right]}\left(Y_{j}\right)$ (recall that $n=h m+(h-1)$ ). Let $a$ and $b$ be two integers such that

$$
t^{a} \cdot \mathbf{B}_{\mathrm{dR}}^{+} \otimes_{F}^{\sigma^{j}} D \subset W_{\mathrm{dR}}^{+, j}(D) \subset t^{-b} \cdot \mathbf{B}_{\mathrm{dR}}^{+} \otimes_{F}^{\sigma^{j}} D,
$$

for all $j$. We then have $\mathrm{M}(D)^{\left[0 ; s_{n}\right]} \subset \lambda^{-b} \cdot \mathcal{R}^{\left[0 ; s_{n}\right]}(Y) \otimes_{F} D$ by Corollary 3.8.

We have $\varphi^{-(h k+j)}\left(\mathcal{R}^{\left[0 ; s_{n}\right]}(Y)\left[1 / f_{j}\right]\right) \subset \mathbf{B}_{\mathrm{dR}}^{+}$for all $1 \leqslant k \leqslant m$ so that if $y \in M_{j}^{\left[0 ; s_{n}\right]}$, then $\varphi^{-(h k+j)}(y) \in W_{\mathrm{dR}}^{+,-j}(D)$ for all $1 \leqslant k \leqslant m$. On the other hand, if $y \in M_{j}^{\left[0 ; s_{n}\right]}$, then $y \in \lambda^{-c} \cdot \mathcal{R}^{\left[0 ; s_{n}\right]}(Y) \otimes_{F} D$ for some $c \geqslant 0$, so that $f_{j}^{a+c} y \in \mathrm{M}(D)^{\left[0 ; s_{n}\right]}$. This implies that $M_{j}^{\left[0 ; s_{n}\right]} \subset \mathrm{M}(D)^{\left[0 ; s_{n}\right]}\left[1 / f_{j}\right]$.

We now prove that $\mathrm{M}(D)^{\left[0 ; s_{n}\right]} \subset M_{j}^{\left[0 ; s_{n}\right]}$. Choose $y \in \mathrm{M}(D)^{\left[0 ; s_{n}\right]}$. Since

$$
\mathrm{M}(D)^{\left[0 ; s_{n}\right]} \subset \lambda^{-b} \cdot \mathcal{R}^{\left[0 ; s_{n}\right]}(Y) \otimes_{F} D,
$$

we can write $y=\lambda^{-b} \sum_{k} z_{k} \otimes d_{k}$. By Weierstrass dividing (Proposition 2.1) the $z_{k}$ 's by the polynomial $\left(Q_{1}\left(Y_{j}\right) \cdots Q_{m}\left(Y_{j}\right)\right)^{a+b}$, we can write $y=\left(Q_{1}\left(Y_{j}\right) \cdots Q_{m}\left(Y_{j}\right)\right)^{a+b} z+y_{0}$ with $y_{0} \in \mathcal{R}^{\left[0 ; s_{n}\right]}(Y)[1 / \lambda] \otimes_{\mathcal{R}^{\left[0 ; s_{n}\right]}\left(Y_{j}\right)} N_{j}^{\left[0 ; s_{n}\right]}$.

Note that $\left(Q_{1}\left(Y_{j}\right) \ldots Q_{m}\left(Y_{j}\right)\right)^{a+b} z \in M_{j}^{\left[0 ; s_{n}\right]}$ because $t^{a} \mathbf{B}_{\mathrm{dR}}^{+} \otimes_{F}^{\sigma^{j}} D \subset W_{\mathrm{dR}}^{+, j}(D)$, so that $\left(Q_{1}\left(Y_{j}\right) \ldots Q_{m}\left(Y_{j}\right)\right)^{a} \cdot D \subset N_{j}^{\left[0 ; s_{n}\right]}$.

Write $y_{0}=\sum_{k=1}^{d} a_{k} \otimes n_{k}$ where $a_{k} \in \mathcal{R}^{\left[0 ; s_{n}\right]}(Y)[1 / \lambda]$ and $n_{1}, \ldots, n_{d}$ is a basis of $N_{j}^{\left[0 ; s_{n}\right]}$. The element $y_{0}$ satisfies $\varphi_{q}^{-\ell} \varphi^{-j}\left(y_{0}\right) \in W_{\mathrm{dR}}^{+,-j}(D)$ for all $1 \leqslant \ell \leqslant m$. By Proposition 5.4, the map

$$
\mathbf{B}_{\mathrm{dR}}^{+} \otimes_{\mathcal{R}^{\left[0 ; s_{n}\right]}\left(Y_{j}\right)}^{\iota_{h \ell+j}} N_{j}^{\left[0 ; s_{n}\right]} \rightarrow W_{\mathrm{dR}}^{+,-j}(D)
$$

is an isomorphism; this implies that $\varphi_{q}^{-\ell} \varphi^{-j}\left(a_{k}\right) \in \mathbf{B}_{\mathrm{dR}}^{+}$for all $1 \leqslant \ell \leqslant m$. Theorem 3.6 now implies that $a_{k}$ has no pole at any of the roots of $Q_{1}\left(Y_{j}\right), \ldots, Q_{m}\left(Y_{j}\right)$, so that 
we have $a_{k} \in \mathcal{R}^{\left[0 ; s_{n}\right]}(Y)\left[1 / f_{j}\right]$. This implies that $y_{0} \in M_{j}^{\left[0 ; s_{n}\right]}$, and therefore also $y$. This proves that $\mathrm{M}(D)^{\left[0 ; s_{n}\right]} \subset M_{j}^{\left[0 ; s_{n}\right]}$ and therefore $\mathrm{M}(D)^{\left[0 ; s_{n}\right]}\left[1 / f_{j}\right]=M_{j}^{\left[0 ; s_{n}\right]}$.

If $x \in \cap_{j} M_{j}^{\left[0 ; s_{n}\right]}$, and if $k=j \bmod h$ with $0 \leqslant j \leqslant h-1$, then the fact that $x \in$ $\mathrm{M}(D)^{\left[0 ; s_{n}\right]}\left[1 / f_{j}\right]=\mathcal{R}^{\left[0 ; s_{n}\right]}(Y)\left[1 / f_{j}\right] \otimes_{\mathcal{R}^{\left[0 ; s_{n}\right]}\left(Y_{j}\right)} N_{j}^{\left[0 ; s_{n}\right]}$ implies that $\iota_{k}(x) \in W_{\mathrm{dR}}^{+,-k}(D)$. This is true for all $h \leqslant k \leqslant n$, so that $x \in \mathrm{M}(D)^{\left[0 ; s_{n}\right]}$ and this proves the second assertion.

Lemma 5.6. We have $\mathrm{M}^{+}(D)\left[1 / f_{j}\right]=\mathcal{R}^{+}(Y)\left[1 / f_{j}\right] \otimes_{\mathcal{R}^{+}\left(Y_{j}\right)} N_{j}^{+}$.

Proof. By combining Propositions 5.4 and 5.5, we find that

$$
\mathrm{M}(D)^{\left[0 ; s_{n}\right]}\left[1 / f_{j}\right]=\mathcal{R}^{\left[0 ; s_{n}\right]}(Y)\left[1 / f_{j}\right] \otimes_{\mathcal{R}^{+}\left(Y_{j}\right)} N_{j}^{+} .
$$

Since $\mathrm{M}(D)^{+}=\cap_{j} \mathrm{M}(D)^{\left[0 ; s_{n}\right]}$, we have $\mathrm{M}(D)^{+}\left[1 / f_{j}\right] \subset \cap_{j} \mathrm{M}(D)^{\left[0 ; s_{n}\right]}\left[1 / f_{j}\right]$. We also have $\mathcal{R}^{+}(Y)\left[1 / f_{j}\right] \otimes_{\mathcal{R}^{+}\left(Y_{j}\right)} N_{j}^{+} \subset \mathrm{M}^{+}(D)\left[1 / f_{j}\right]$, and those two inclusions imply that $\mathrm{M}^{+}(D)\left[1 / f_{j}\right]=\mathcal{R}^{+}(Y)\left[1 / f_{j}\right] \otimes_{\mathcal{R}^{+}\left(Y_{j}\right)} N_{j}^{+}$.

Proof of Theorem 5.3. We first prove that the family $\left\{\mathrm{M}(D)^{\left[0 ; s_{n}\right]}\right\}_{n \in S}$ is a coherent sheaf. Take $n \geqslant m \in S$. We have

$$
\begin{aligned}
\mathcal{R}^{\left[0 ; s_{m}\right]}(Y) & \otimes_{\mathcal{R}^{\left[0 ; s_{n}\right]}(Y)} \mathrm{M}(D)^{\left[0 ; s_{n}\right]} \\
& =\mathcal{R}^{\left[0 ; s_{m}\right]}(Y) \otimes_{\mathcal{R}^{\left[0 ; s_{n}\right]}(Y)}\left(\cap_{j} \mathcal{R}^{\left[0 ; s_{n}\right]}(Y)\left[1 / f_{j}\right] \otimes_{\mathcal{R}^{\left[0 ; s_{n}\right]}\left(Y_{j}\right)} N_{j}^{\left[0 ; s_{n}\right]}\right) \\
& =\cap_{j} \mathcal{R}^{\left[0 ; s_{m}\right]}(Y)\left[1 / f_{j}\right] \otimes_{\mathcal{R}^{\left[0 ; s_{n}\right]}\left(Y_{j}\right)} N_{j}^{\left[0 ; s_{n}\right]}=\mathrm{M}(D)^{\left[0 ; s_{m}\right]} .
\end{aligned}
$$

This implies that the family $\left\{\mathrm{M}(D)^{\left[0 ; s_{n}\right]}\right\}_{n \in S}$ is a coherent sheaf. It is clear that its global sections are precisely $\mathrm{M}^{+}(D)$. By Proposition 5.5, we have $\mathrm{M}(D)^{\left[0 ; s_{n}\right]}=$ $\cap_{j} \mathrm{M}(D)^{\left[0 ; s_{n}\right]}\left[1 / f_{j}\right]$ where each $\mathrm{M}(D)^{\left[0 ; s_{n}\right]}\left[1 / f_{j}\right]$ is free of rank $d$ over $\mathcal{R}(Y)^{\left[0 ; s_{n}\right]}\left[1 / f_{j}\right]$. The fact that $\mathrm{M}(D)^{\left[0 ; s_{n}\right]}$ is reflexive now follows from Proposition 6 of VII.4.2 of [8], and this proves (1).

By combining Proposition 5.4 and Lemma 5.6, we get item (2) of the theorem. Suppose now that $x \in \cap_{j} \mathrm{M}^{+}(D)\left[1 / f_{j}\right]$. If $k=j \bmod h$ with $0 \leqslant j \leqslant h-1$, then the fact that $x \in \mathrm{M}^{+}(D)\left[1 / f_{j}\right]=\mathcal{R}^{+}(Y)\left[1 / f_{j}\right] \otimes_{\mathcal{R}^{+}\left(Y_{j}\right)} N_{j}^{+}$implies that $\iota_{k}(x) \in$ $W_{\mathrm{dR}}^{+,-k}(D)$. This being true for all $k \geqslant h$, we have $x \in \mathrm{M}^{+}(D)$ and this proves item (3) of the theorem.

Remark 5.7. If $h \leqslant 2$, then the ring $\mathcal{R}^{\left[0 ; s_{n}\right]}(Y)$ is of dimension $\leqslant 2$, and reflexive $\mathcal{R}^{\left[0 ; s_{n}\right]}(Y)$-modules are therefore projective. By Lütkebohmert's theorem (see [19], corollary on page 128), the $\mathcal{R}^{\left[0 ; s_{n}\right]}(Y)$-module $\mathrm{M}(D)^{\left[0 ; s_{n}\right]}$ is then free of rank $d$. The system $\left\{\mathrm{M}(D)^{\left[0 ; s_{n}\right]}\right\}_{n \in S}$ then forms a vector bundle over the open unit polydisk. By combining Proposition 2 on page 87 of [16] (note that " $A_{m}$ " is defined at the bottom of page 82 of loc. cit.), and the main theorem of [1], we get that $\mathrm{M}^{+}(D)$ is free of rank $d$ over $\mathcal{R}^{+}(Y)$. If $h \geqslant 3$, I do not know whether this still holds.

\section{Properties of $\mathrm{M}^{+}(D)$}

We now prove that $\mathrm{M}(D)=\mathcal{R}(Y) \otimes_{\mathcal{R}^{+}(Y)} \mathrm{M}^{+}(D)$ is a $\left(\varphi_{q}, \Gamma_{F}\right)$-module over $\mathcal{R}(Y)$, and that if $D$ arises from a crystalline representation $V$, then $\mathrm{M}^{+}(D)$ and $V$ are naturally 
related. It is clear from the definition that $\mathrm{M}^{+}(D)$ is stable under the action of $\Gamma_{F}$. We also have $\lambda^{a} \cdot \mathcal{R}^{+}(Y) \otimes_{F} D \subset \mathrm{M}^{+}(D)$ for some $a \geqslant 0$, so that

$$
\mathcal{R}^{+}(Y)[1 / \lambda] \otimes_{\mathcal{R}^{+}(Y)} \mathrm{M}^{+}(D)=\mathcal{R}^{+}(Y)[1 / \lambda] \otimes_{F} D .
$$

Say that the module $D$ with $h$ filtrations is effective if $\operatorname{Fil}_{j}^{0}(D)=D$ for $0 \leqslant j \leqslant h-1$. Recall that $n=h m+(h-1)$ with $m \geqslant 1$.

Lemma 6.1. If $D$ is effective, then the $\mathcal{R}^{+}\left(Y_{j}\right)$-module $N_{j}^{+}$is stable under $\varphi_{q}$, and $N_{j}^{+} / \varphi_{q}^{*}\left(N_{j}^{+}\right)$is killed by $Q_{1}\left(Y_{j}\right)^{a_{j}}$ if $a_{j} \geqslant 0$ is such that $\mathrm{Fil}^{a_{j}+1} D=\{0\}$.

Proof. This concerns the construction in one variable, so the proof is standard. See for example Section 2.2 of [18].

Proposition 6.2. If $D$ is effective, then the $\mathcal{R}^{+}(Y)$-module $\mathrm{M}^{+}(D)$ is stable under the Frobenius map $\varphi_{q}$, and $\mathrm{M}^{+}(D) / \varphi_{q}^{*}\left(\mathrm{M}^{+}(D)\right)$ is killed by $Q_{1}\left(Y_{0}\right)^{a_{0}} \cdots Q_{1}\left(Y_{h-1}\right)^{a_{h-1}}$.

Proof. By (2) of Theorem 5.3, we have $\mathrm{M}^{+}(D)=\cap_{j} \mathrm{M}^{+}(D)\left[1 / f_{j}\right]$ and by Lemma 5.6, $\mathrm{M}^{+}(D)\left[1 / f_{j}\right]=\mathcal{R}^{+}(Y)\left[1 / f_{j}\right] \otimes_{\mathcal{R}^{+}\left(Y_{j}\right)} N_{j}^{+}$. Lemma 6.1 implies that $N_{j}^{+}$is stable under $\varphi_{q}$, and so the same is true of $\mathrm{M}^{+}(D)\left[1 / f_{j}\right]$ and hence $\mathrm{M}^{+}(D)$.

If $x \in \mathrm{M}^{+}(D)$, then $x \in \mathrm{M}^{+}(D)\left[1 / f_{j}\right]=\mathcal{R}^{+}(Y)\left[1 / f_{j}\right] \otimes_{\mathcal{R}^{+}\left(Y_{j}\right)} N_{j}^{+}$. Note however that at each $k=i \neq j \bmod h$, the coefficients of $x$ can have a pole of order at most $a_{i}$ since $\mathrm{Fil}^{a_{i}+1} D=\{0\}$. This implies the more precise estimate

$$
\mathrm{M}^{+}(D) \subset \prod_{i \neq j} \lambda_{i}^{-a_{i}} \cdot \mathcal{R}^{+}(Y) \otimes_{\mathcal{R}^{+}\left(Y_{j}\right)} N_{j}^{+} .
$$

The $\varphi_{q}\left(\mathcal{R}^{+}(Y)\right)$-module $\mathcal{R}^{+}(Y)$ is free of rank $q^{h}$, with basis $\left\{Y^{\ell}, \ell \in\{0, \ldots, q-1\}^{h}\right\}$. We therefore have

$$
\begin{aligned}
Q_{1}\left(Y_{0}\right)^{a_{0}} \cdots Q_{1}\left(Y_{h-1}\right)^{a_{h-1}} \cdot x & \in \prod_{i \neq j}\left(\lambda_{i} / Q_{1}\left(Y_{i}\right)\right)^{-a_{i}} \cdot \mathcal{R}^{+}(Y) \otimes_{\mathcal{R}^{+}\left(Y_{j}\right)} Q_{1}\left(Y_{j}\right)^{a_{j}} \cdot N_{j}^{+} \\
& \subset \oplus_{\ell} Y^{\ell} \cdot \varphi_{q}\left(\mathcal{R}^{+}(Y)\left[1 / f_{j}\right] \otimes_{\mathcal{R}^{+}\left(Y_{j}\right)} N_{j}^{+}\right) .
\end{aligned}
$$

This implies that

$$
Q_{1}\left(Y_{0}\right)^{a_{0}} \cdots Q_{1}\left(Y_{h-1}\right)^{a_{h-1}} \cdot x \in \cap_{j} \oplus_{\ell} Y^{\ell} \cdot \varphi_{q}\left(\mathrm{M}^{+}(D)\left[1 / f_{j}\right]\right)=\varphi_{q}^{*}\left(\mathrm{M}^{+}(D)\right),
$$

which proves the second claim.

Remark 6.3. Instead of working with a $D$ where the filtrations are defined on $D$, we could have asked for the filtrations to be defined on $F_{n} \otimes_{F} D$ for some $n \geqslant 1$. The construction and properties of $\mathrm{M}^{+}(D)$ are then basically unchanged, but the annihilator of $\mathrm{M}^{+}(D) / \varphi_{q}^{*}\left(\mathrm{M}^{+}(D)\right)$ is possibly more complicated than in Proposition 6.2. This applies in particular to representations of $G_{F}$ that become crystalline when restricted to $G_{F_{n}}$ for some $n \geqslant 1$.

Definition 6.4. A $\left(\varphi_{q}, \Gamma_{F}\right)$-module over $\mathcal{R}(Y)$ is a $\mathcal{R}(Y)$-module $\mathrm{M}$ that is of the form $\mathrm{M}=\mathcal{R}(Y) \otimes_{\mathcal{R}^{[s ;+\infty}[(Y)} \mathrm{M}^{[s ;+\infty[}$ where $\mathrm{M}^{[s ;+\infty[}$ is a coadmissible $\mathcal{R}^{[s ;+\infty[}(Y)$ module, endowed with a semilinear Frobenius map $\varphi_{q}: \mathrm{M}^{[s ;+\infty[} \rightarrow \mathrm{M}^{[q s ;+\infty[}$, such that $\varphi_{q}^{*}\left(\mathrm{M}^{[s ;+\infty[}\right)=\mathrm{M}^{[q s ;+\infty[}$, and a continuous and compatible action of $\Gamma_{F}$. 
Remark 6.5. In the definition above, it would seem natural to impose some additional condition on M, such as "torsion-free." All the $\left(\varphi_{q}, \Gamma_{F}\right)$-modules over $\mathcal{R}(Y)$ that are constructed in this article are actually reflexive. The definition above should be considered provisional, until we have a better idea of which objects we want to exclude. Note that in the absence of flatness, tensor products may behave badly.

If $D$ is a $\varphi_{q}$-module with an action of $\Gamma_{F}$ and $h$ filtrations and if $\ell \in \mathbf{Z}$, let $D(\ell)$ denote the same $\varphi_{q}$-module with an action of $\Gamma_{F}$, but with $\operatorname{Fil}_{j}^{k}(D(\ell))=\left(\operatorname{Fil}_{j}^{k-\ell} D\right)(\ell)$. Note that $D(\ell)$ is effective if $\ell \gg 0$.

Lemma 6.6. We have $\mathrm{M}(D(\ell))=\lambda^{-\ell} \cdot \mathrm{M}(D)$.

Proof. The fact that $\mathrm{M}^{+}(D(\ell))=\lambda^{-\ell} \cdot \mathrm{M}^{+}(D)$ follows from the definition.

Theorem 6.7. If $D$ is a $\varphi_{q}$-module with an action of $\Gamma_{F}$ and $h$ filtrations as above, then $\mathcal{R}(Y) \otimes_{\mathcal{R}^{+}(Y)} \mathrm{M}^{+}(D)$ is a $\left(\varphi_{q}, \Gamma_{F}\right)$-module over $\mathcal{R}(Y)$.

Proof. If $D$ is effective, then this follows from Theorem 5.3 and Proposition 6.2. If $D$ is not effective, then $D(\ell)$ is effective if $\ell \gg 0$, and the theorem follows from the effective case and Lemma 6.6.

Remark 6.8. In [18], Kisin and Ren construct some $\left(\varphi_{q}, \Gamma_{F}\right)$-modules $\mathrm{M}_{\mathrm{KR}}^{+}(D)$ in one variable, over the ring $\mathcal{R}^{+}\left(Y_{0}\right)$, from the data of a $D$ like ours for which the filtration Fil $_{j}^{\bullet}$ is trivial for $j \neq 0$. For those $D$, we have $\mathrm{M}^{+}(D)=\mathcal{R}^{+}(Y) \otimes_{\mathcal{R}^{+}\left(Y_{0}\right)} \mathrm{M}_{\mathrm{KR}}^{+}(D)$. More generally, our construction shows that $\mathrm{M}^{+}(D)$ comes by extension of scalars from a $\left(\varphi_{q}, \Gamma_{F}\right)$-module in as many variables as there are non-trivial filtrations among the $\mathrm{Fil}_{j}^{\bullet}$.

Proposition 6.9. If $n=h k+j \geqslant h$, then the map

$$
\mathbf{B}_{\mathrm{dR}}^{+} \otimes_{\mathcal{R}^{+}(Y)}^{\iota_{n}} \mathrm{M}^{+}(D) \rightarrow \mathrm{Fil}_{-j}^{0}\left(\mathbf{B}_{\mathrm{dR}} \otimes_{F}^{\sigma^{-j}} D\right)
$$

is an isomorphism.

Proof. Since $\iota_{n}\left(f_{j}\right)$ is a unit of $\mathbf{B}_{\mathrm{dR}}^{+}$, we have

$$
\begin{aligned}
\mathbf{B}_{\mathrm{dR}}^{+} \otimes_{\mathcal{R}^{+}(Y)}^{\iota_{n}} \mathrm{M}^{+}(D) & =\mathbf{B}_{\mathrm{dR}}^{+} \otimes_{\mathcal{R}^{+}(Y)\left[1 / f_{j}\right]}^{\iota_{n}} \mathrm{M}^{+}(D)\left[1 / f_{j}\right] \\
& =\mathbf{B}_{\mathrm{dR}}^{+} \otimes_{\mathcal{R}^{+}\left(Y_{j}\right)}^{\iota_{n}} N_{j}^{+} \\
& =\mathrm{Fil}_{-j}^{0}\left(\mathbf{B}_{\mathrm{dR}} \otimes_{F}^{\sigma^{-j}} D\right),
\end{aligned}
$$

where the last equality follows from Proposition 5.4.

Suppose now that $D$ comes from an $F$-linear crystalline representation $V$ of $G_{F}$ as in Example 5.1. In this case, $\operatorname{Fil}_{j}^{0}\left(\mathbf{B}_{\mathrm{dR}} \otimes_{F}^{\sigma^{j}} D\right)=\mathbf{B}_{\mathrm{dR}}^{+} \otimes_{F}^{\sigma^{j}} V$. Moreover, one recovers $V$ from $D$ by the formula:

$$
V=\left\{y \in\left(\widetilde{\mathbf{B}}_{\mathrm{rig}}^{+}[1 / t] \otimes_{F} D\right)^{\varphi_{q}=1}, \iota_{j}(y) \in \mathrm{Fil}_{-j}^{0}\left(\mathbf{B}_{\mathrm{dR}} \otimes_{F}^{\sigma^{-j}} D\right) \text { for all } 0 \leqslant j \leqslant h-1\right\} .
$$

Recall that we have constructed in Section 3 an injective map $\mathcal{R}^{+}(Y) \rightarrow \widetilde{\mathbf{B}}_{\text {rig. }}^{+}$. This way we get a map

$$
\widetilde{\mathbf{B}}_{\text {rig }}^{+} \otimes_{\mathcal{R}^{+}(Y)} \mathrm{M}^{+}(D) \rightarrow \widetilde{\mathbf{B}}_{\text {rig }}^{+}[1 / t] \otimes_{F} D \rightarrow \widetilde{\mathbf{B}}_{\text {rig }}^{+}[1 / t] \otimes_{F} V .
$$

Let $\widetilde{\mathbf{B}}_{\text {rig }}^{\dagger, r}$ be the rings defined in Section 2.3 [2]. Recall that $n(r)$ is the smallest $n$ such that $r \leqslant p^{n-1}(p-1)$. We have the following lemma. 
Lemma 6.10. If $y \in \widetilde{\mathbf{B}}_{\text {rig }}^{\dagger, r}[1 / t]$ satisfies $\varphi^{-n}(y) \in \mathbf{B}_{\mathrm{dR}}^{+}$for all $n \geqslant n(r)$, then $y \in \widetilde{\mathbf{B}}_{\mathrm{rig}}^{\dagger, r}$. Proof. See Lemma 1.1 of [5] and the proof of Proposition 3.2 in ibid.

Theorem 6.11. If $D$ comes from a crystalline representation $V$, and if $r \geqslant p^{h-1}(p-$ $1)$, then the map above gives rise to an isomorphism

$$
\widetilde{\mathbf{B}}_{\text {rig }}^{\dagger, r} \otimes_{\mathcal{R}^{+}(Y)} \mathrm{M}^{+}(D) \rightarrow \widetilde{\mathbf{B}}_{\text {rig }}^{\dagger, r} \otimes_{F} V .
$$

Proof. We first check that the image of the map above belongs to $\widetilde{\mathbf{B}}_{\text {rig }}^{\dagger, r} \otimes_{F} V$. If $y \in \widetilde{\mathbf{B}}_{\text {rig }}^{\dagger, r} \otimes_{\mathcal{R}^{+}(Y)} \mathrm{M}^{+}(D)$, then its image is in $\widetilde{\mathbf{B}}_{\text {rig }}^{\dagger, r}[1 / t] \otimes_{F} V$ and satisfies $\varphi^{-n}(y) \in$ $\mathbf{B}_{\mathrm{dR}}^{+} \otimes_{F}^{\sigma^{-n}} V$ for all $n \geqslant n(r)$, so the assertion follows from Lemma 6.10 .

We now prove that $\widetilde{\mathbf{B}}_{\text {rig }}^{\dagger, r} \otimes_{\mathcal{R}^{+}(Y)} \mathrm{M}^{+}(D)$ is a free $\widetilde{\mathbf{B}}_{\text {rig }}^{\dagger, r}$-module of rank $d$. By (2) of Theorem 5.3, $\mathrm{M}^{+}(D)\left[1 / f_{j}\right]$ is a free $\mathcal{R}^{+}(Y)\left[1 / f_{j}\right]$-module of rank $d$, and therefore $\widetilde{\mathbf{B}}_{\text {rig }}^{\dagger, r}\left[1 / f_{j}\right] \otimes_{\mathcal{R}^{+}(Y)} \mathrm{M}^{+}(D)$ is a free $\widetilde{\mathbf{B}}_{\text {rig }}^{\dagger, r}\left[1 / f_{j}\right]$-module of rank $d$ for all $j$. The ring $\widetilde{\mathbf{B}}_{\text {rig }}^{\dagger, r}$ is a Bézout ring by Theorem 2.9.6 of [17], and the elements $f_{0}, \ldots, f_{h-1}$ have no common factor. They therefore generate the unit ideal of $\widetilde{\mathbf{B}}_{\text {rig }}^{\dagger, r}$, and $\widetilde{\mathbf{B}}_{\text {rig }}^{\dagger, r} \otimes_{\mathcal{R}^{+}(Y)} \mathrm{M}^{+}(D)$ is projective of rank $d$ by Theorem 1 of II.5.2 of [8]. Since $\widetilde{\mathbf{B}}_{\text {rig }}^{\dagger, r}$ is a Bézout ring, $\widetilde{\mathbf{B}}_{\text {rig }}^{\dagger, r} \otimes_{\mathcal{R}^{+}(Y)} \mathrm{M}^{+}(D)$ is free of rank $d$. By Proposition 6.9, the map

$$
\mathbf{B}_{\mathrm{dR}}^{+} \otimes_{\widetilde{\mathbf{B}}_{\mathrm{rig}}^{\dagger, r}}^{\iota_{n}}\left(\widetilde{\mathbf{B}}_{\mathrm{rig}}^{\dagger, r} \otimes_{\mathcal{R}^{+}(Y)} \mathrm{M}^{+}(D)\right) \rightarrow \mathbf{B}_{\mathrm{dR}}^{+} \otimes_{F}^{\sigma^{-n}} V
$$

is an isomorphism if $n \geqslant n(r)$. The two $\widetilde{\mathbf{B}}_{\text {rig }}^{\dagger, r}$-modules $\widetilde{\mathbf{B}}_{\text {rig }}^{\dagger, r} \otimes_{\mathcal{R}^{+}(Y)} \mathrm{M}^{+}(D)$ and $\widetilde{\mathbf{B}}_{\text {rig }}^{\dagger, r} \otimes_{F}$ $V$ therefore have the same localizations at all $n \geqslant n(r)$, and are both stable under $G_{F}$, so that they are equal by the same argument as in the proof of Lemma 2.2.2 of [3] (the idea is to take determinants, so that one is reduced to showing that if $x \in \widetilde{\mathbf{B}}_{\text {rig }}^{\dagger, r}$ generates an ideal stable under $G_{F}$, and has the property that $\iota_{n}(x)$ is a unit of $\mathbf{B}_{\mathrm{dR}}^{+}$ for all $n \geqslant n(r)$, then $x$ is a unit of $\widetilde{\mathbf{B}}_{\text {rig }}^{\dagger, r}$ ).

Remark 6.12. If $D$ comes from a crystalline representation $V$, and if $n \geqslant 0$, then there is likewise an isomorphism $\widetilde{\mathbf{B}}_{\text {rig }}^{\dagger, r} \otimes_{\mathcal{R}^{+}(Y)}^{\varphi^{-n}} \mathrm{M}^{+}(D) \rightarrow \widetilde{\mathbf{B}}_{\text {rig }}^{\dagger, r} \otimes_{F}^{\sigma^{-n}} V$ for $r \gg 0$.

\section{Crystalline $\left(\varphi_{q}, \Gamma_{F}\right)$-modules}

Let $\mathrm{M}$ be a $\left(\varphi_{q}, \Gamma_{F}\right)$-module over $\mathcal{R}(Y)$. In this section, we define what it means for $\mathrm{M}$ to be crystalline, and we prove that every crystalline $\left(\varphi_{q}, \Gamma_{F}\right)$-module $\mathrm{M}$ is of the form $\mathrm{M}=\mathrm{M}(D)$, where $D$ is a $\varphi_{q}$-module with $h$ filtrations, on which the action of $G_{F}$ is trivial. The results are similar to those of [4], which deals with the cyclotomic case.

Lemma 7.1. We have $\operatorname{Frac}(\mathcal{R}(Y))^{\Gamma_{F}}=F$.

Proof. If $x \in \operatorname{Frac}(\mathcal{R}(Y))^{\Gamma_{F}}$, then we can write $x=a / b$ with $a, b \in \mathcal{R}^{\left[s_{n} ; s_{n}\right]}(Y)$ for some $n \gg 0$. By Proposition 3.2, the series $a\left(u, \ldots, \varphi^{h-1}(u)\right)$ and $b\left(u, \ldots, \varphi^{h-1}(u)\right)$ converge in $\widetilde{\mathbf{B}}^{\left[r_{n} ; r_{n}\right]}$. We can therefore see $\varphi^{-n}(a)$ and $\varphi^{-n}(b)$ as elements of $\mathbf{B}_{\mathrm{dR}}^{+}$, which satisfy $\varphi^{-n}(a) / \varphi^{-n}(b) \in \mathbf{B}_{\mathrm{dR}}^{G_{F}}$. The lemma now follows from the fact that $\mathbf{B}_{\mathrm{dR}}^{G_{F}}=F$.

If $\mathrm{M}$ is a $\left(\varphi_{q}, \Gamma_{F}\right)$-module over $\mathcal{R}(Y)$, then let $\mathrm{D}_{\text {cris }}(\mathrm{M})=\left(\mathcal{R}(Y)[1 / t] \otimes_{\mathcal{R}(Y)} \mathrm{M}\right)^{\Gamma_{F}}$. 
Corollary 7.2. If $\mathrm{M}$ is a $\left(\varphi_{q}, \Gamma_{F}\right)$-module over $\mathcal{R}(Y)$, then we have $\operatorname{dim} \mathrm{D}_{\text {cris }}(\mathrm{M}) \leqslant$ $\operatorname{dim} \operatorname{Frac}(\mathcal{R}(Y)) \otimes_{\mathcal{R}(Y)} \mathrm{M}$.

Proof. By a standard argument, Lemma 7.1 implies that the map

$$
\operatorname{Frac}(\mathcal{R}(Y)) \otimes_{F} \mathrm{D}_{\text {cris }}(V) \rightarrow \operatorname{Frac}(\mathcal{R}(Y)) \otimes_{\mathcal{R}(Y)} \mathrm{M}
$$

is injective.

Definition 7.3. We say that a $\left(\varphi_{q}, \Gamma_{F}\right)$-module $\mathrm{M}$ over $\mathcal{R}(Y)$ is crystalline if

(1) for some $s, \mathrm{M}^{[s ;+\infty}\left[1 / f_{j}\right]$ is a free $\mathcal{R}(Y)^{[s ;+\infty[}\left[1 / f_{j}\right]$-module of finite rank $d$;

(2) $\mathrm{M}^{[s ;+\infty[}=\cap_{j=0}^{h-1} \mathrm{M}^{[s ;+\infty[}\left[1 / f_{j}\right]$;

(3) we have $\operatorname{dim} \mathrm{D}_{\text {cris }}(\mathrm{M})=d$.

For example, if $D$ is a $\varphi_{q}$-module with $h$ filtrations on which the action of $G_{F}$ is trivial, then $\mathrm{M}(D)$ is a crystalline $\left(\varphi_{q}, \Gamma_{F}\right)$-module. Note that a crystalline $\left(\varphi_{q}, \Gamma_{F}\right)$ module is reflexive.

Proposition 7.4. If $f \in \mathcal{R}^{[s ;+\infty[}(Y)$ generates an ideal of $\mathcal{R}^{[s ;+\infty[}(Y)$ that is stable under $\Gamma_{F}$, then $f=u \cdot \prod_{j=0}^{h-1} \prod_{n \geqslant n(s)}\left(Q_{n}\left(Y_{j}\right) / p\right)^{a_{n, j}}$ where $u$ is a unit and $a_{n, j} \in \mathbf{Z}_{\geqslant 0}$.

Proof. Recall that a power series $f \in \mathcal{R}^{I}(Y)$ is a unit if and only if it has no zero in the corresponding domain of convergence (by the nullstellensatz, see Section 7.1.2 of [7]).

Let $I=[s ; u]$ be a closed subinterval of $\left[s ;+\infty\left[\right.\right.$, so that $f \in \mathcal{R}^{I}(Y)$, and let $z=\left(z_{0}, z_{1}, \ldots, z_{h-1}\right)$ be a point such that $f(z)=0$. Let $J$ be the set of indices $j$ such that $z_{j}$ is not a torsion point of $\mathrm{LT}_{h}$ and let $f_{J} \in \mathcal{R}_{F_{k}}^{I}\left(\left\{Y_{j}\right\}_{j \in J}\right)$ be the power series that results from evaluation of the $Y_{m}$ at $z_{m}$ for all the $z_{m}$ that are torsion points of $\mathrm{LT}_{h}$ (here $k$ is large enough so that all those $z_{m}$ belong to $F_{k}$ ). The ideal of $\mathcal{R}_{F_{k}}^{I}\left(\left\{Y_{j}\right\}_{j \in J}\right)$ generated by the power series $f_{J}$ is stable under $1+p^{k} \mathcal{O}_{F}$, so that the set of its zeroes is stable under the action of $1+p^{k} \mathcal{O}_{F}$. Furthermore, $f_{J}$ has a zero none of whose coordinates are torsion points of $\mathrm{LT}_{h}$. The same argument as in the proof of Proposition 2.4 shows that $f_{J}=0$.

If we denote by $Z_{I}(f)$ the zero set of $f \in \mathcal{R}^{I}(Y)$, then the preceding argument shows that $Z_{I}(f)$ is the union of finitely many components of the form $Z_{0} \times \cdots \times Z_{h-1}$ where for each $j$, either $Z_{j}$ is a torsion point of $\mathrm{LT}_{h}$ or $Z_{j}=Z_{I}(\{0\})$. For reasons of dimension, each of these components has precisely one $Z_{j}$ which is a torsion point, the remaining $h-1$ being $Z_{I}(\{0\})$. This implies that in $\mathcal{R}^{I}(Y), f$ is the product of finitely many $Q_{n}\left(Y_{j}\right)$ by a unit.

The proposition now follows by a standard infinite factorisation argument, by writing $\left[s ;+\infty\left[=\cup_{u \geqslant s}[s ; u]\right.\right.$.

Corollary 7.5. If $\mathrm{M}$ is a crystalline $\left(\varphi_{q}, \Gamma_{F}\right)$-module over $\mathcal{R}(Y)$, then the map

$$
\mathcal{R}(Y)[1 / t] \otimes_{F} \mathrm{D}_{\text {cris }}(\mathrm{M}) \rightarrow \mathcal{R}(Y)[1 / t] \otimes_{\mathcal{R}(Y)} \mathrm{M}
$$

is an isomorphism.

Proof. The map is injective by Lemma 7.1, and its determinant generates an ideal of $\mathcal{R}(Y)[1 / t]$ that is stable under $\Gamma_{F}$. Proposition 7.4 implies that this ideal is the unit ideal of $\mathcal{R}(Y)[1 / t]$, and therefore that the map is an isomorphism.

We now consider filtrations on $\mathrm{D}_{\text {cris }}(\mathrm{M})$. 
Lemma 7.6. Let $D$ be an F-vector space, and let $W$ be a $\mathbf{B}_{\mathrm{dR}}^{+}$-lattice of $\mathbf{B}_{\mathrm{dR}} \otimes_{F} D$ that is stable under $G_{F}$, where $G_{F}$ acts trivially on $D$. If we set $\mathrm{Fil}^{i} D=D \cap t^{i} \cdot W$, then $W=\operatorname{Fil}^{0}\left(\mathbf{B}_{\mathrm{dR}} \otimes_{F} D\right)$.

Proof. Let $e_{1}, \ldots, e_{d}$ be a basis of $D$ adapted to its filtration, with $e_{i} \in \mathrm{Fil}^{h_{i}} \backslash \mathrm{Fil}^{h_{i}+1} D$. We then have $\mathrm{Fil}^{0}\left(\mathbf{B}_{\mathrm{dR}} \otimes_{F} D\right)=\oplus_{i=1}^{d} \mathbf{B}_{\mathrm{dR}}^{+} \cdot t^{-h_{i}} e_{i}$. By definition, we have $t^{-h_{i}} e_{i} \in W$, so that $\operatorname{Fil}^{0}\left(\mathbf{B}_{\mathrm{dR}} \otimes_{F} D\right) \subset W$. We now prove the reverse inclusion.

If $w \in W$, then we can write $w=a_{1} t^{-h_{1}} e_{1}+\cdots+a_{d} t^{-h_{d}} e_{d}$ with $a_{i} \in \mathbf{B}_{\mathrm{dR}}$ and we need to prove that $a_{i} \in \mathbf{B}_{\mathrm{dR}}^{+}$for all $i$. If this is not the case, then there exists $n \geqslant 1$ such that if we set $b_{i}=t^{n} a_{i}$, then we have $b_{1} t^{-h_{1}} e_{1}+\cdots+b_{d} t^{-h_{d}} e_{d} \in t \cdot W$, with $b_{i} \in\left(\mathbf{B}_{\mathrm{dR}}^{+}\right)^{\times}$for at least one $i$. Consider the shortest such relation; in particular, $b_{i} \in\left(\mathbf{B}_{\mathrm{dR}}^{+}\right)^{\times}$for all $i$ for which $b_{i} \neq 0$, and we can assume that $b_{i}=1$ for at least one $i$. If $g \in G_{F}$, then applying $1-\chi_{\text {cyc }}(g)^{h_{i}} g$ to the relation yields a shorter relation. This implies that $\left(1-\chi_{\text {cyc }}(g)^{h_{i}-h_{j}} g\right)\left(b_{j}\right) \in t \mathbf{B}_{\mathrm{dR}}^{+}$for all $g \in G_{F}$ and all $1 \leqslant j \leqslant d$. Since $H^{0}\left(G_{F}, \mathbf{C}_{p}\right)=F$ and $H^{0}\left(G_{F}, \mathbf{C}_{p}(h)\right)=\{0\}$ if $h \neq 0$, we have $b_{j} \in F+t \mathbf{B}_{\mathrm{dR}}^{+}$if $h_{i}=h_{j}$ and $b_{j} \in t \mathbf{B}_{\mathrm{dR}}^{+}$otherwise. The relation above therefore reduces to an $F$-linear combination of those $e_{j}$ for which $h_{j}=h_{i}$, belonging to $D \cap t^{h_{i}+1} W=\mathrm{Fil}^{h_{i}+1} D$, and is hence trivial. This proves that $W \subset \operatorname{Fil}^{0}\left(\mathbf{B}_{\mathrm{dR}} \otimes_{F} D\right)$.

Definition 7.7. Let $\mathrm{M}$ be a crystalline $\left(\varphi_{q}, \Gamma_{F}\right)$-module over $\mathcal{R}(Y)$. For $m \gg 0$ and $j=0, \ldots, h-1$ and $n=h m-j$, define

$\operatorname{Fil}_{j}^{i}\left(F \otimes_{F}^{\sigma^{j}} \varphi_{q}^{-m}\left(\mathrm{D}_{\text {cris }}(\mathrm{M})\right)\right)=\left(F \otimes_{F}^{\sigma^{j}} \varphi_{q}^{-m}\left(\mathrm{D}_{\text {cris }}(\mathrm{M})\right)\right) \cap t^{i} \cdot\left(\mathbf{B}_{\mathrm{dR}}^{+} \otimes_{\mathcal{R}^{[s ;+\infty[(Y)}}^{\varphi^{-n}} \mathrm{M}^{[s ;+\infty[}\right)$.

Proposition 7.8. The definition of $\mathrm{Fil}_{j}^{i}\left(\mathrm{D}_{\text {cris }}(\mathrm{M})\right)$ does not depend on $m \gg 0$, and we have $\operatorname{Fil}^{0}\left(\mathbf{B}_{\mathrm{dR}} \otimes_{F}^{\sigma^{-n}} \mathrm{D}_{\text {cris }}(\mathrm{M})\right)=\mathbf{B}_{\mathrm{dR}}^{+} \otimes_{\mathcal{R}^{[s ;+\infty[(Y)}}^{\varphi^{-n}} \mathrm{M}^{[s ;+\infty[}$.

Proof. If $s$ is large enough, then $\mathrm{M}^{[q s ;+\infty[}=\varphi_{q}^{*}\left(\mathrm{M}^{[s ;+\infty[}\right)$ so that

$\mathbf{B}_{\mathrm{dR}}^{+} \otimes_{\mathcal{R}[q s+\infty[(Y)}^{\varphi^{-n-h}} \mathrm{M}^{[q s ;+\infty[}=\mathbf{B}_{\mathrm{dR}}^{+} \otimes_{\mathcal{R}[q s ;+\infty[(Y)}^{\varphi^{-n} \varphi_{q}^{-1}} \varphi_{q}^{*}\left(\mathrm{M}^{[s ;+\infty[}\right)=\mathbf{B}_{\mathrm{dR}}^{+} \otimes_{\mathcal{R}[s ;+\infty[(Y)}^{\varphi^{-n}} \mathrm{M}^{[s ;+\infty[}$,

which implies the first statement. The second statement follows from Lemma 7.6, applied to $W=\mathbf{B}_{\mathrm{dR}}^{+} \otimes_{\mathcal{R}^{[s ;+\infty[(Y)}}^{\varphi^{-n}} \mathrm{M}^{[s ;+\infty[}$.

Theorem 7.9. The functors $\mathrm{M} \mapsto \mathrm{D}_{\text {cris }}(\mathrm{M})$ and $D \mapsto \mathrm{M}(D)$, between the category of crystalline $\left(\varphi_{q}, \Gamma_{F}\right)$-modules over $\mathcal{R}(Y)$ and the category of $\varphi_{q}$-modules with $h$ filtrations, are mutually inverse.

Proof. If $D$ is a $\varphi_{q}$-module with $h$ filtrations, then it is clear that $\mathrm{D}_{\text {cris }}(\mathrm{M}(D))=D$ as $\varphi_{q}$-modules. The fact that $\operatorname{Fil}_{j}^{i}(D)=D \cap t^{i} \cdot \operatorname{Fil}_{j}^{0}\left(\mathbf{B}_{\mathrm{dR}} \otimes_{F}^{\sigma^{-n}} D\right)$ follows from taking a basis of $D$ adapted to $\mathrm{Fil}_{j}^{\bullet}$ and

$$
\operatorname{Fil}_{j}^{0}\left(\mathbf{B}_{\mathrm{dR}} \otimes_{F}^{\sigma^{-n}} D\right)=\mathbf{B}_{\mathrm{dR}}^{+} \otimes_{\mathcal{R}^{[s ;+\infty[(Y)}}^{\varphi^{-n}} \mathrm{M}^{[s ;+\infty[}(D)=\mathrm{Fil}_{j}^{0}\left(\mathbf{B}_{\mathrm{dR}} \otimes_{F}^{\sigma^{-n}} \mathrm{D}_{\text {cris }}(\mathrm{M}(D))\right)
$$

by Propositions 6.9 and 7.8, so that the filtrations on $D$ and $\mathrm{D}_{\text {cris }}(\mathrm{M})$ are the same.

We now check that if $\mathrm{M}$ is a crystalline $\left(\varphi_{q}, \Gamma_{F}\right)$-module over $\mathcal{R}(Y)$ and $D=\mathrm{D}_{\text {cris }}(\mathrm{M})$ with the filtration given in Definition 7.7, then $\mathrm{M}=\mathrm{M}(D)$. Corollary 7.5 says that we have $\mathcal{R}(Y)[1 / t] \otimes_{F} D=\mathcal{R}(Y)[1 / t] \otimes_{\mathcal{R}(Y)}$ M. The theorem now follows

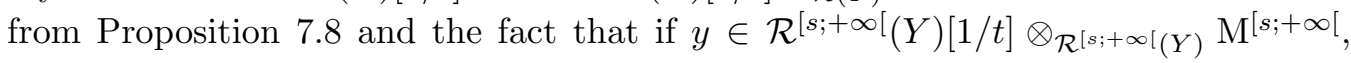




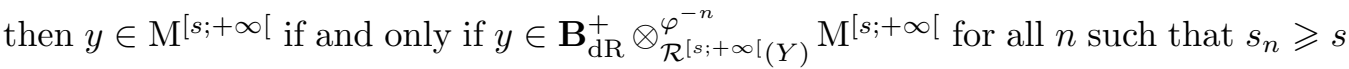
by Corollary 3.8 and items (1) and (2) of Definition 7.3.

\section{Acknowledgments}

I am grateful to P. Colmez, J.-M. Fontaine, L. Fourquaux, M. Gros, K. Kedlaya, R. Liu, V. Pilloni, S. Rozensztajn, P. Schneider, B. Stroh, L. Xiao, B. Xie, S. Zerbes and especially C. Breuil for helpful conversations and remarks. Special thanks to P. Schneider and the referee for pointing out several embarassing mistakes in a previous version of this paper and helping me to correct them. This research is partially supported by the ANR grant ThéHopaD (Théorie de Hodge $p$-adique et Développements) ANR-11-BS01-005.

\section{References}

[1] W. Bartenwerfer, k-holomorphe Vektorraumbündel auf offenen Polyzylindern, J. Reine Angew. Math. 326 (1981), 214-220.

[2] L. Berger, Représentations p-adiques et équations différentielles, Invent. Math. 148(2) (2002), 219-284.

[3] - Construction de $(\varphi, \Gamma)$-modules: représentations p-adiques et B-paires, Algeb. Number Theory 2(1) (2008), 91-120.

[4] — Equations différentielles p-adiques et $(\varphi, N)$-modules filtrés, Astérisque (319) (2008), $13-38$.

[5] — Presque $\mathbf{C}_{p}$-représentations et $(\varphi, \Gamma)$-modules, J. Inst. Math. Jussieu 8(4) (2009), 653-668.

[6] L. Berger and P. Colmez, Théorie de Sen et vecteurs localement analytiques (2013), Preprint available at: perso.ens-lyon.fr/laurent.berger/prepublications.php.

[7] S. Bosch, U. Güntzer and R. Remmert, Non-Archimedean analysis, Grundlehren der Mathematischen Wissenschaften, 261 Springer-Verlag, Berlin (1984), ISBN 3-540-12546-9.

[8] N. Bourbaki, Éléments de mathématique. Algèbre commutative, Actualités Scientifiques et Industrielles, Hermann, Paris, 1961.

[9] C. Breuil, The emerging p-adic Langlands programme, in 'Proceedings of the International Congress of Mathematicians', volume II, 203-230, Hindustan Book Agency, New Delhi, 2010.

[10] P. Colmez, Espaces de Banach de dimension finie, J. Inst. Math. Jussieu 1(3) (2002), 331-439.

$[11] \longrightarrow,(\varphi, \Gamma)$-modules et représentations du mirabolique de $\mathrm{GL}_{2}\left(\mathbf{Q}_{p}\right)$, Astérisque (330) (2010), $61-153$.

[12] J.-M. Fontaine, Représentations p-adiques des corps locaux. I, in The Grothendieck Festschrift, Vol. II, Vol. 87 of Progr. Math., 249-309, Birkhäuser Boston, Boston, MA, 1990.

[13] — Le corps des périodes p-adiques, Astérisque (223) (1994), 59-111. With an appendix by Pierre Colmez, Périodes $p$-adiques (Bures-sur-Yvette, 1988).

[14] J.-M. Fontaine and J.-P. Wintenberger, Le "corps des normes" de certaines extensions algébriques de corps locaux, C. R. Acad. Sci. Paris Sér. A-B 288(6) (1979), A367-A370.

[15] L. Fourquaux and B. Xie, Triangulable $\mathcal{O}_{F}$-analytic $\left(\varphi_{q}, \Gamma\right)$-modules of rank 2 , Algeb. Number Theory (to appear)

[16] L. Gruson, Fibrés vectoriels sur un polydisque ultramétrique, Ann. Sci. École Norm. Sup. (4) 1 (1968), 45-89.

[17] K.S. Kedlaya, Slope filtrations revisited, Doc. Math. 10 (2005), 447-525 (electronic).

[18] M. Kisin and W. Ren, Galois representations and Lubin-Tate groups, Doc. Math. 14 (2009), 441-461.

[19] W. Lütkebohmert, Vektorraumbündel über nichtarchimedischen holomorphen Räumen, Math. Z. 152(2) (1977), 127-143.

[20] P. Schneider and J. Teitelbaum, p-adic Fourier theory, Doc. Math. 6 (2001), 447-481 (electronic). 
[21] - Algebras of $p$-adic distributions and admissible representations, Invent. Math. 153(1) (2003), 145-196.

[22] J.-P. Wintenberger, Le corps des normes de certaines extensions infinies de corps locaux; applications, Ann. Sci. École Norm. Sup. (4) 16(1) (1983), 59-89.

[23] G. Zábrádi, Generalized Robba rings, Israel J. Math. 191 (2012), 817-887. With an appendix by Peter Schneider.

UMPA ENS DE LYON, UMR 5669 DU CNRS, IUF

E-mail address: laurent.berger@ens-lyon.fr

$U R L$ : perso.ens-lyon.fr/laurent.berger/ 